\title{
Receptivity of Hypersonic Boundary Layers to Acoustic and Vortical Disturbances
}

\author{
P. Balakumar ${ }^{*}$ and Michael A. Kegerise* \\ Flow Physics and Control Branch \\ NASA Langley Research Center, Hampton, VA 23581
}

\begin{abstract}
Boundary layer receptivity to two-dimensional acoustic disturbances at different incidence angles and to vortical disturbances is investigated by solving the Navier-Stokes equations for Mach 6 flow over a $7^{\circ}$ half-angle sharp-tipped wedge and a cone. Higher order spatial and temporal schemes are employed to obtain the solution. The results show that the instability waves are generated in the leading edge region and that the boundary layer is much more receptive to slow acoustic waves as compared to the fast waves. It is found that the receptivity of the boundary layer on the 'windward' side (with respect to the acoustic forcing) decreases when the incidence angle is increased from 0 to 30 degrees. However, the receptivity coefficient for the 'leeward' side is found to vary relatively weakly with the incidence angle. The maximum receptivity is obtained when the wave incident angle is about 20 degrees. Vortical disturbances also generate unstable second modes, however the receptivity coefficients are smaller than that for the acoustic waves. Vortical disturbances first generate the fast acoustic modes and they switch to the slow mode near the continuous spectrum.
\end{abstract}

\section{Introduction}

$\mathrm{T}$ RANSITION in hypersonic boundary layers in quiet environments occurs due to the growth of unstable second mode disturbances inside the boundary layer ${ }^{1}$. Numerical, experimental, and theoretical investigations have been performed to try to understand these instability modes for hypersonic boundary layers ${ }^{2-19}$. Stability characteristics of a boundary layer depend on several parameters including: boundary layer edge Mach number (M), local Reynolds number, pressure gradient, and wall conditions. The instability is governed by the linearized Navier-Stokes equations. The normal mode analysis of this equation yields an eigenvalue system that determines the stability characteristics of the boundary layer. The solution of this system yields several discrete and continuous eigensolutions. The eigensolutions corresponding to the continuous spectrum give the local forced response of the boundary layer to incoming or outgoing acoustic, vortical, or entropy disturbances. Without any local forcing, the contribution of the continuous spectrum to the boundary layer disturbances does not exist. Among the discrete eigensolutions, only one or a few, at most, may be unstable. These unstable modes grow exponentially downstream and cause the boundary layer to transition to turbulence.

In hypersonic boundary layers, among all the discrete modes, the slow and the fast modes are the two relevant modes for boundary layer transition ${ }^{12,13}$. The slow and the fast modes correspond to the discrete modes whose phase speeds approach the phase speeds of the slow and the fast acoustic wave, $1-1 / M$ and $1+1 / M$, respectively, as the Reynolds number decreases towards zero. As the Reynolds number increases, the phase speed of the slow mode increases gradually from $1-1 / M$ to 1.0 and the phase speed of the fast mode decreases gradually from $1+1 / M$ to 1.0 . As the phase speed of the fast mode approaches 1.0, the fast mode is absorbed into the continuous spectrum. Downstream of this point, a new mode with slightly different eigenvalues and eigenfunctions springs from the continuous spectrum. Even though there is a discontinuity in the eigenvalues and eigenfunctions across the continuous spectrum, the new mode is continued to be termed as the fast mode. Further downstream, the phase speed of the fast mode continues to decrease and at some point the phase speeds of the slow, and the fast modes cross each other. This point is termed as the synchronization point. However, the imaginary parts of the modes remain separate at this point.

\footnotetext{
${ }^{*}$ Research Scientist, Member AIAA

${ }^{* *}$ Research Scientist, Member AIAA
} 
Depending on the frequency, Mach number, and wall conditions, one of the modes, either the slow or the fast mode evolves into Mack's unstable second mode, and the other mode evolves into the stable mode. Gushin et al. (1991) and Fedorov et al. (2001) developed an analytical model using a quadratic dispersion relation in the wavenumber and Reynolds number plane to explain the observed branching of the slow and the fast modes into stable and unstable modes. The boundary layer characteristics have been computed by many researchers without much difficulty. The important relevant and unanswered questions are: (1) How are the slow and the fast modes generated inside the boundary layers in wind tunnel and flight environments? (2) What are the initial amplitudes of these modes in a particular environment? (3) How and where do the slow and/or fast modes generate the unstable second mode? (4) What is the initial amplitude of the second mode?

Building on the fact that the phase speeds of the slow and fast acoustic waves synchronize with the phase speeds of the slow and fast modes, several investigations were conducted on the interaction of acoustic waves with supersonic and hypersonic boundary layers. Fedorov and Khokhlov ${ }^{14,15,16}$ showed using asymptotic theory that eigen solutions are formed near the leading edge region of a flat plate during the diffraction of the acoustic wave by the growing boundary layer. This diffraction zone is very long in the streamwise direction on the order of $\varepsilon^{-2} \lambda$ in the streamwise direction, where $\varepsilon$ is an asymptotically small parameter and $\lambda$ is the wavelength of the free stream acoustic disturbances. Fedorov [2003] also identified that the unstable second mode can be generated through the inter-modal exchange between the fast and the slow modes near the synchronization point. For an adiabatic wall, Fedorov [2003] found that receptivity to slow acoustic waves could be as much as 50 times the receptivity to the fast acoustic waves. Thus, the leading-edge receptivity via the slow mode excitation is much stronger than in the case of inter-modal exchange.

Ma and Zhong ${ }^{18,19}$ have performed a series of direct numerical simulations for a flow over a sharp Mach 4.5 flatplate boundary layer to investigate receptivity to fast acoustic waves. They used a fifth-order accurate shock-fitting method in the numerical simulation. They identified from the wavenumber and the eigenfucntions comparison with the linear stability calculations that the fast stable mode excited the unstable Mack's second mode through the mechanism of inter-modal exchange. They also considered the effects of incidence angles on the receptivity of the supersonic boundary layer to fast acoustic waves. The results showed that maximum receptivity of the second mode is obtained when the incident wave angle approximately equals $26^{\circ}$.

In previous work ${ }^{20-24}$, the interactions of slow and fast acoustic waves with hypersonic boundary layers over sharp and blunt flat plates, wedges, and cones were investigated. The results show that flows over geometries with sharp leading edges or tips produce instability waves, which originate very close to the leading edge or tip and have receptivity coefficients about 5 to 10 times the amplitude of the forced slow acoustic wave. It was also found that the amplitude of the instability waves generated by the slow acoustic waves is about 20 times larger than that for fast acoustic waves. In addition, it was observed that bluntness and wall cooling stabilize the first-mode disturbances and the initial amplitudes of the instability waves are several orders of magnitude smaller than the amplitude of the forced acoustic waves.

Most of the freestream acoustic field in wind tunnels is associated with the radiated noise from the nozzle wall. The nozzle wall boundary layer whether turbulent or laminar radiates the noise at an angle to the free stream. Most of our previous computations were performed for plane acoustic waves and in this paper we consider the effects of the acoustic incidence angle on the receptivity of instability waves in hypersonic boundary layers. In flight environments, however, these acoustic fields likely do not exist or dominate the disturbance field. The question then arises as to how these small-wavelength $(\sim \mathrm{mm})$, high-frequency unstable disturbances are generated inside the boundary layers. One possible source may be the atmospheric turbulence. In this paper, we investigate the effects of (1) acoustic wave incidence angles and (2) vorticity waves on the receptivity of instability waves in hypersonic boundary layers over a $7^{\circ}$ half-angle sharp-tipped wedge and a cone at a free stream Mach number of 6 .

\section{Models and Flow Conditions}

The models are a $7^{\circ}$ half-angle sharp-tipped wedge and a cone with a nose radius of $0.015 \mathrm{~mm}$ (Fig. 1). Computations were performed for a freestream Mach number of 6 . The freestream stagnation temperature and pressure were $430^{\circ} \mathrm{K}$ and $12.2 \mathrm{kPa}(140 \mathrm{psi})$, yielding a unit Reynolds number of $10.4^{*} 10^{6} / \mathrm{m}$. The simulations were performed for a constant wall temperature of $300^{\circ} \mathrm{K}$. The cone model geometry was tested in the Boeing/AFOSR Mach 6 Quiet Tunnel at the same freestream conditions. ${ }^{9}$ The flow parameters and the formulae that were employed are given in Table 1. 
Table 1. Flow parameters used in this study.

Freestream Mach number $M_{\infty}=6.0$

Free stream Reynolds number $R e_{\infty}=10.4 * 10^{6} / \mathrm{m}$

Free stream density $\rho_{\infty}=4.062 * 10^{-2} \mathrm{~kg} / \mathrm{m}^{3}$

Free stream pressure $p_{\infty}=611.36 \mathrm{~N} / \mathrm{m}^{2}$

Free stream velocity $U_{\infty}=870.9 \mathrm{~m} / \mathrm{s}$

Free stream temperature $T_{\infty}=52.44^{\circ} \mathrm{K}$

Sutherland viscosity formulae $\mu=1.458 * 10^{-6} T^{1.5} /(T+110.4) \mathrm{kg} / \mathrm{m} / \mathrm{s}$

Free stream kinematic viscosity $v_{\infty}=8.369 * 10^{-4} \mathrm{~m}^{2} / \mathrm{s}$

Wall temperature $=300{ }^{\circ} \mathrm{K}$

Prandtl number $P r=0.70$

Ratio of specific heats $\gamma=1.4$

Length scale $\sqrt{\frac{v_{\infty} x_{0}}{U_{\infty}}}=9.8032 * 10^{-5} \mathrm{~m} .\left(x_{0}=0.1 \mathrm{~m}\right.$. $)$

The non-dimensional frequency $F$ is defined as $F=\frac{2 \pi v_{\infty} f}{U_{\infty}^{2}}$,

where $f$ is the frequency in Hertz. For the above flow conditions, $F=1 * 10^{-4}$ corresponds to a frequency of $144.23 \mathrm{kHz}$.

\section{Governing Equations}

The governing equations are the two-dimensional unsteady compressible Navier-Stokes equations, written in conservation form and in cylindrical coordinates:

$$
\begin{gathered}
\frac{\partial}{\partial t} r Q+\frac{\partial r F}{\partial x}+\frac{\partial r G}{\partial r}=S, \\
Q=\left[\begin{array}{l}
\rho \\
\rho E \\
\rho u \\
\rho v
\end{array}\right] .
\end{gathered}
$$

Here, $(x, r)$ are the cylindrical coordinates, $(u, v)$ are the velocity components, $\rho$ is the density, and $p$ is the pressure. The total energy, $E$, is given by:

$$
\begin{gathered}
E=e+\frac{u^{2}+v^{2}}{2}, \\
e=c_{v} T, p=\rho R T,
\end{gathered}
$$


where $e$ is the internal energy and $T$ is the temperature. The fluxes $F, G$, and the source term $S$ are described in Kara et $a .^{21}$ The viscosity, $\mu$, is computed using Sutherland's law and the thermal conductivity, $k$, is given in terms of the Prandtl number, $P r$. The variables $\rho, p, T$ and velocity are non-dimensionalized by their corresponding reference variables $\rho_{\infty} p_{\infty}, T_{\infty}$ and $\sqrt{R T_{\infty}}$ respectively. The reference value for length is given by $\sqrt{v x_{0} / U_{\infty}}$, where $x_{0}$ is a reference location. For the computations, the equations were transformed from the physical coordinate system $(x, r)$ to the computational curvilinear coordinate system $(\xi, \eta)$ in a conservative manner.

\section{A. Solution Algorithm}

The governing equations were solved using a $5^{\text {th }}$-order accurate weighted essentially non-oscillatory (WENO) scheme for space discretization and a $3^{\text {rd }}$-order total-variation-diminishing (TVD) Runge-Kutta scheme for time integration. The WENO and TVD methods and formulas are explained in $\mathrm{Shu}^{25}$. The application of the ENO method to the Navier-Stokes equations is presented by Atkins ${ }^{26}$. The solution method implemented in the present computations is described in Balakumar ${ }^{27}$.

A schematic diagram of the computational setup is shown in Fig. 1. The outer boundary of the computational domain lies outside the shock and follows a parabola so that the boundary layer growth was accurately captured. At the outflow boundary of the computational domain, an extrapolation boundary condition was used. At the wall, the simulations employed viscous conditions for the velocities and a constant wall temperature for the temperature. The density was computed from the continuity equation. In the mean-flow computations, the free-stream values at the outer boundary were prescribed. In all cases, the model centerline was aligned with the freestream flow. In the unsteady computations, acoustic or vortical perturbations were superimposed on the uniform mean flow at the outer boundary of the computational domain.

The solution procedure was to first compute the steady mean flow by performing unsteady computations using a variable time step until the maximum residual reaches a small value $\left(\sim 10^{-11}\right)$. For those computations, a CFL number of 0.2 was used. The next steps were (1) to introduce unsteady acoustic or vortical disturbances at the outer boundary of the computational domain and (2) to perform time accurate simulations to investigate the interaction of these disturbances with the boundary layer and their subsequent downstream evolution. Linearized Euler equations in a uniform mean flow are solved in Cartesian and cylindrical coordinates to obtain analytical expressions for the acoustic and vortical disturbances that were to be superimposed at the outer boundary. Since we use a very fine spatial grid to resolve the leading-edge region, these computations require a very small time step, which is determined by the minimum time step allowed by a CFL number of 0.2 . In the nose region, about 400,000 time steps per cycle are used in the simulation. The computational domain is divided into two regions and larger time steps are taken in the downstream region. While the simulation allows nonlinear effects, the forcing amplitude is kept small enough in this study for dominant mechanisms to be governed by linear dynamics.

The computational grid, which was generated using analytical formulae, stretches in the $\eta$ direction close to the wall and is uniform outside of the boundary layer. In the $\xi$ direction, the grid was very fine near the nose and then uniform in the region downstream. The outer boundary that lays outside of the shock follows a parabola with its vertex located a short distance upstream of the leading edge of the plate. The computational domain extends from $x$ $=-0.001$ to 0.5 meters in the axial direction. This gives a maximum Reynolds number of $R e=2280.0$ at the end of the computational domain. Calculations were performed using a grid size of $7001 * 251$.

\section{Plane waves.}

The linearized Euler equations in Cartesian coordinates in a uniform mean flow are: 


$$
\begin{aligned}
& \frac{\partial \rho}{\partial t}+U_{0} \frac{\partial \rho}{\partial x}+\rho_{0} \frac{\partial u}{\partial x}+\rho_{0} \frac{\partial v}{\partial y}+\rho_{0} \frac{\partial w}{\partial z}=0, \\
& \rho_{0} \frac{\partial u}{\partial t}+\rho_{0} U_{0} \frac{\partial u}{\partial x}=-\frac{\partial p}{\partial x}, \\
& \rho_{0} \frac{\partial v}{\partial t}+\rho_{0} U_{0} \frac{\partial v}{\partial x}=-\frac{\partial p}{\partial y}, \\
& \rho_{0} \frac{\partial w}{\partial t}+\rho_{0} U_{0} \frac{\partial w}{\partial x}=-\frac{\partial p}{\partial z}, \\
& \rho_{0} c_{p} \frac{\partial T}{\partial t}+\rho_{0} U_{0} c_{p} \frac{\partial T}{\partial x}=\frac{\partial p}{\partial t}+U_{0} \frac{\partial p}{\partial x}, \\
& P_{0}=\rho_{0} R T_{0}, \\
& p=\rho_{0} R T+\rho R T_{0} .
\end{aligned}
$$

(a) Acoustic waves.

The solution of this system for an acoustic wave can be written as

$$
\left\{\begin{array}{l}
\rho \\
u \\
v \\
w \\
T
\end{array}\right\}=\left\{\begin{array}{c}
\frac{1}{a_{0}^{2}} \\
-\frac{\alpha_{a c}}{\rho_{0}\left(\alpha_{a c} U_{0}-\omega\right)} \\
-\frac{\varepsilon_{a c}}{\rho_{0}\left(\alpha_{a c} U_{0}-\omega\right)} \\
-\frac{\beta_{a c}}{\rho_{0}\left(\alpha_{a c} U_{0}-\omega\right)} \\
\frac{(\gamma-1) T_{0}}{\rho_{0} a_{0}^{2}}
\end{array}\right\} p .
$$

Here the pressure $p$ is in the form

$$
p=p_{a m p} e^{i\left(\alpha_{a c} x+\varepsilon_{a c} y+\beta_{a c} z-\omega t\right)} .
$$

The dispersion relation among the wavenumbers $\alpha_{a c}, \beta_{a c}, \varepsilon_{a c}$ and the frequency $\omega$ is given by

$$
\left(\alpha_{a c} U_{0}-\omega\right)^{2}=\left(\alpha_{a c}{ }^{2}+\beta_{a c}{ }^{2}+\varepsilon_{a c}{ }^{2}\right) a_{0}^{2} .
$$

For acoustic disturbances with zero sweeps (i.e., $\beta_{a c}=0$ ), the $x$-wavenumber $\alpha_{\mathrm{ac}}$ can be expressed as

$$
\alpha_{a c}=\frac{\omega \cos \theta_{y}}{\left(U_{0} \cos \theta_{y} \pm a_{0}\right)} .
$$

Here $\theta_{y}=\tan ^{-1}\left(\frac{\varepsilon_{a c}}{\alpha_{a c}}\right)$ is the incident angle and for $\varepsilon_{a c}<0$ the plate is radiated from above and for $\varepsilon_{a c}>0$ the plate is radiated from below or represents the wave which is reflected from the plate. The plus sign corresponds to the fast 
moving wave and the minus sign corresponds to the slow moving wave. The corresponding phase speeds are $C=U_{0} \pm \frac{a_{0}}{\cos \theta_{y}}$.

\section{(b) Vorticity waves.}

The solution of the linearized Euler equations for the vorticity waves can be written as

$$
\left\{\begin{array}{c}
\rho \\
u \\
v \\
w \\
T
\end{array}\right\}=\left\{\begin{array}{c}
0 \\
\tilde{u} \\
\tilde{v} \\
\tilde{w} \\
0
\end{array}\right\} e^{i\left(\alpha_{v} x+\varepsilon_{v} y+\beta_{v} z-\omega t\right)}
$$

The dispersion relation among the wavenumbers $\alpha_{v}, \beta_{v}, \varepsilon_{v}$ and the frequency $\omega$ is given by

$$
\left(\alpha_{v} U_{0}-\omega\right)=0
$$

The continuity equation imposes the following constrain on the velocity components

$$
\tilde{u} \alpha_{v}+\tilde{v} \varepsilon_{v}+\tilde{w} \beta_{v}=0
$$

Computations are performed for a two-dimensional vortical disturbance with

$$
\begin{aligned}
& \tilde{u}=0 \\
& \varepsilon_{v}=0 \\
& \beta_{v}=0
\end{aligned}
$$

\section{Circular waves.}

The linearized Euler equations in cylindrical coordinates in a uniform mean flow are:

$$
\begin{aligned}
& \frac{\partial \rho}{\partial t}+U_{0} \frac{\partial \rho}{\partial x}+\rho_{0} \frac{\partial u}{\partial x}+\rho_{0} \frac{\partial v}{\partial r}+\frac{\rho_{0}}{r} \frac{\partial w}{\partial \theta}+\rho_{0} \frac{v}{r}=0, \\
& \rho_{0} \frac{\partial u}{\partial t}+\rho_{0} U_{0} \frac{\partial u}{\partial x}=-\frac{\partial p}{\partial x}, \\
& \rho_{0} \frac{\partial v}{\partial t}+\rho_{0} U_{0} \frac{\partial v}{\partial x}=-\frac{\partial p}{\partial y}, \\
& \rho_{0} \frac{\partial w}{\partial t}+\rho_{0} U_{0} \frac{\partial w}{\partial x}=-\frac{1}{r} \frac{\partial p}{\partial \theta}, \\
& \rho_{0} c_{p} \frac{\partial T}{\partial t}+\rho_{0} U_{0} c_{p} \frac{\partial T}{\partial x}=\frac{\partial p}{\partial t}+U_{0} \frac{\partial p}{\partial x}, \\
& P_{0}=\rho_{0} R T_{0}, \\
& p=\rho_{0} R T+\rho R T_{0} .
\end{aligned}
$$




\section{(a) Acoustic waves.}

The solution of this system for an acoustic wave can be written as

$$
\left\{\begin{array}{l}
\rho \\
u \\
v \\
w \\
T
\end{array}\right\}=\left\{\begin{array}{c}
\frac{1}{a_{0}^{2}} p \\
-\frac{\alpha_{a c}}{\rho_{0}\left(\alpha_{a c} U_{0}-\omega\right)} p \\
-\frac{1}{i \rho_{0}\left(\alpha_{a c} U_{0}-\omega\right)} \frac{\partial p}{\partial r} \\
-\frac{m}{r \rho_{0}\left(\alpha_{a c} U_{0}-\omega\right)} p \\
\frac{(\gamma-1) T_{0}}{\rho_{0} a_{0}^{2}} p
\end{array}\right\} .
$$

Here the pressure $p$ is in the form

$$
p=p_{a m p} J_{m}\left(\alpha_{n} r\right) e^{i\left(\alpha_{a c} x+m \theta-\omega t\right)} .
$$

where, $J_{m}$ is the Bessel function of order $m$. The dispersion relation among the wavenumber $\alpha_{a c}$ and the frequency $\omega$ is given by

$$
a_{0}^{2} \alpha_{n}^{2}=\left(\alpha_{a c} U_{0}-\omega\right)^{2}-\alpha_{a c}^{2} a_{0}^{2}
$$

\section{(b) Vorticity waves.}

The solution of the linearized Euler equations in cylindrical coordinates for the vorticity waves can be written as

$$
\left\{\begin{array}{c}
\rho \\
u \\
v \\
w \\
T
\end{array}\right\}=\left\{\begin{array}{c}
0 \\
\tilde{u}(r) \\
\tilde{v}(r) \\
\tilde{w}(r) \\
0
\end{array}\right\} e^{i\left(\alpha_{v} x+m \theta-\omega t\right)}
$$

The dispersion relation among the wavenumber $\alpha_{v}$ and the frequency $\omega$ is given by

$$
\left(\alpha_{v} U_{0}-\omega\right)=0
$$

The continuity equation imposes the following constrain on the velocity components

$$
\frac{\partial \tilde{v}}{\partial r}+\frac{\tilde{v}}{r}+i \tilde{u} \alpha_{v}+\frac{i m}{r} \tilde{w}=0
$$

Computations are performed for a two-dimensional vortical disturbance with 


$$
\begin{aligned}
& \tilde{v}=-\frac{1}{2} i \tilde{u} r \\
& m=0
\end{aligned}
$$

Table 2. Values of $\alpha_{\text {ac }}$ and wavelength for different incidence angles at the frequency of $F=0.95 * 10^{-4}$ for the

\begin{tabular}{|c|c|c|c|c|c|c|c|}
\hline \multirow[t]{2}{*}{$\theta_{\mathrm{y}}$} & \multirow{2}{*}{$\begin{array}{c}\text { Slow } \\
\text { acoustic }\end{array}$} & \multirow{2}{*}{$\begin{array}{c}\text { Fast } \\
\text { acoustic }\end{array}$} & \multirow[t]{2}{*}{ Vorticity } & \multicolumn{2}{|c|}{$\begin{array}{c}\text { Wedge( Stability) } \\
\text { X=20mm }\end{array}$} & \multicolumn{2}{|c|}{$\begin{array}{c}\text { Cone (Stability) } \\
\text { X=60mm }\end{array}$} \\
\hline & & & & Slow & Fast & Slow & Fast \\
\hline 0 & $\begin{array}{c}0.1162 \\
(5.29 \mathrm{~mm})\end{array}$ & $\begin{array}{c}0.0830 \\
(7.41 \mathrm{~mm})\end{array}$ & $\begin{array}{c}0.097 \\
(6.35 \mathrm{~mm})\end{array}$ & $\begin{array}{c}0.119 \\
(5.16 \mathrm{~mm})\end{array}$ & $\begin{array}{c}0.083 \\
(7.41 \mathrm{~mm})\end{array}$ & & \\
\hline $\begin{array}{c}0 \\
\text { Cone }\end{array}$ & $\begin{array}{c}0.1835 \\
(3.35 \mathrm{~mm})\end{array}$ & $\begin{array}{c}0.1310 \\
(4.69 \mathrm{~mm})\end{array}$ & $\begin{array}{c}0.153 \\
(4.02 \mathrm{~mm})\end{array}$ & & & $\begin{array}{c}0.1772 \\
(3.47 \mathrm{~mm})\end{array}$ & $\begin{array}{c}0.1328 \\
(4.64 \mathrm{~mm})\end{array}$ \\
\hline 10 & $\begin{array}{c}0.1166 \\
(5.28 \mathrm{~mm})\end{array}$ & $\begin{array}{c}0.0828 \\
(7.43 \mathrm{~mm})\end{array}$ & & & & & \\
\hline 20 & $\begin{array}{c}0.1178 \\
(5.23 \mathrm{~mm})\end{array}$ & $\begin{array}{c}0.0823 \\
(7.48 \mathrm{~mm})\end{array}$ & & & & & \\
\hline 30 & $\begin{array}{c}0.1200 \\
(5.13 \mathrm{~mm})\end{array}$ & $\begin{array}{c}0.0812 \\
(7.58 \mathrm{~mm})\end{array}$ & & & & & \\
\hline $\begin{array}{c}\text { Axi. Sym } \\
\alpha_{n}=0.05\end{array}$ & $\begin{array}{c}0.185 \\
(3.33 \mathrm{~mm})\end{array}$ & $\begin{array}{c}0.130 \\
(4.74 \mathrm{~mm})\end{array}$ & & & & $\begin{array}{c}0.1772 \\
(3.47 \mathrm{~mm})\end{array}$ & $\begin{array}{c}0.1328 \\
(4.64 \mathrm{~mm})\end{array}$ \\
\hline
\end{tabular}
wedge and at the frequency of $F=1.5 * 10^{-4}$ for the cone.

Table 2 gives the wavenumbers and the wavelengths in dimensional and non-dimensional units for the slow and the fast acoustic waves, the vorticity waves and for the neutral stability waves for the wedge and the cone. It is interesting to see that in all the cases the wavelengths for the acoustic waves match with the wavelengths for the stability waves. The wavelengths of the slow and the fast acoustic waves at zero incidence at the frequency $F=$ $0.95 * 10^{-4}$ are 5.29 and $7.41 \mathrm{~mm}$, and the wavelengths for the slow and the fast instability waves near the leading edge of the wedge are 5.16 and $7.41 \mathrm{~mm}$. Similarly for the cone, they are $(3.35,4.69)$ and $(3.47,4.64)$. The wavelengths of the vorticity waves do not match the wavelengths of either the slow or the fast modes. The wavelength of the vorticity wave at the frequency of $F=0.95 * 10^{-4}$ is $6.35 \mathrm{~mm}$ which lies in the middle of the wavelengths for the slow and the fast stability modes.

\section{Results}

\section{A. $7^{\circ}$ Half-Angle Sharp-Tipped Wedge}

\section{Mean Flow}

Figure 2 presents the computed mean density contours for the sharp-tipped wedge. The flow field for the entire computational domain is shown in Fig. 2, while the insert shows a close-up view of the nose region. The leading edge shock is located approximately at $0.01 \mathrm{~mm}$. upstream of the leading edge. The boundary layer edge values obtained from the simulation are compared in Table 3 with the results obtained from the shock relations for a wedge. The computed results are almost the same as that obtained from the inviscid analysis. 
$49^{\text {th }}$ AIAA Aerosciences Meeting, January 4-7, 2011, Orlando, Florida

Table 3. Comparison of conditions at the edge of the boundary layer with the inviscid solution downstream of the shock.

\begin{tabular}{|c|c|c|c|c|c|c|}
\hline $\begin{array}{c}\text { Wedge } \\
\text { angle }\end{array}$ & Shock angle & $\begin{array}{c}\text { Mach } \\
\text { number }\end{array}$ & $\begin{array}{c}\text { Pressure } \\
\text { ratio }\end{array}$ & $\begin{array}{c}\text { Density } \\
\text { ratio }\end{array}$ & $\begin{array}{c}\text { Temperature } \\
\text { ratio }\end{array}$ & $\begin{array}{c}\text { Unit } \\
\text { Reynolds } \\
\text { number } \\
\text { ratio }\end{array}$ \\
\hline $\begin{array}{c}7 \\
\text { Inviscid }\end{array}$ & 14.838 & 5.048 & 2.587 & 1.924 & 1.344 & 1.34 \\
\hline $\begin{array}{c}7 \\
\text { Simulation }\end{array}$ & 15.0 & 5.03 & 2.60 & 1.94 & 1.35 & \\
\hline
\end{tabular}

The density profiles at $x=1.0,3.0,10.0,20.030 .0$ and $50.0 \mathrm{~cm}$. are plotted in Fig. 3(a). Figure 3(b) shows the same profiles in the similarity coordinates. The compressible Blasius similarity profile is also included for comparison. The boundary layer profiles slowly approach the Blasius similarity profile close to $x=50 \mathrm{~cm}$. The boundary layer thickness near $x=50 \mathrm{~cm}$ is about $3 \mathrm{~mm}$.

\section{Linear stability}

Linear stability results for the mean flow profiles obtained from the simulation are presented in Figs. 4 and 5. Figure 4(a) shows the growth rate for different frequencies $F=0.85^{*} 10^{-4}, 0.90^{*} 10^{-4}, 0.95^{*} 10^{-4}$, and $1.0^{*} 10^{-4}$ while Fig. 4(b) depicts the N-Factor curves for these frequencies. The growth rate curves exhibit a long region of neutrally stable Mack's first mode followed by a narrow strongly unstable second mode region. The unstable second mode region shifts downstream with decreasing frequency. The maximum N-Factor reached within the computational domain of $x=50 \mathrm{~cm}$ is about 4.0. The most amplified frequency is $F=0.95 * 10^{-4}$.

Figures 5(a) and 5(b) show the evolution of the eigenvalues along the axial direction for the slow (Mode $\mathrm{S}$ ) and fast (Mode F) modes for the frequency $F=0.95^{*} 10^{-4}$. Figure 5(a) shows the evolution of phase speed $c_{r}$ and the growth rate $-\alpha_{i}$, and Fig. 5(b) shows the wavenumber $\alpha_{r}$ and the wavelength. The slow mode or the Mode $\mathrm{S}$ synchronizes with the slow acoustic wave with the phase speed of $(U-a)$ or $1-1 / M$ as the Reynolds number or $x$ becomes small. The fast mode or the Mode F synchronizes with the fast acoustic wave with the phase speed of $(U+a)$ or $1+1 / M$ as the Reynolds number becomes small. The phase speed of the slow mode increases from $1-1 / M$ to 1.0 as the Reynolds number increases while the phase speed of the fast mode decreases from $1+1 / M$ to a smaller value. As the fast mode evolves downstream, it merges with the continuous spectrum with phase speed $c_{r}=1.0$. For this frequency of $F=0.95^{*} 10^{-4}$ the merging occurs around $x=281 \mathrm{~mm}$. Downstream of the continuous spectrum a new mode with slightly different eigenvalues and eigenfunctions emerges. These points are also marked as red dots in Fig. 5(a). Hence, the fast mode has a discontinuity across the continuous spectrum. This may have some effects in the receptivity process of generating the unstable second mode. As the slow mode and the new fast mode evolve downstream, the slow mode becomes the unstable Mack's second mode and the fast mode becomes the stable mode. Another observation is that the phase speeds of the slow mode and the fast mode coalesce near $x=400 \mathrm{~mm}$. However, the growth rates of the slow mode and the fast mode at this point are quite different. The growth rates are $-\alpha_{i}=0.005$ and -0.01 for the slow and the fast modes respectively. This point is also marked as a line in Figs 5(a) and (b). Fedorov (2001) called this point the synchronization point for the fast and the slow mode and claims that near this point inter-modal energy exchange could occur depending on the Mach number, frequency, and the wall conditions. He developed an analytical model using a quadratic dispersion relation to explain the observed growth rate pattern in hypersonic boundary layers. The important question is what happens when a fast mode evolves downstream? Does it generate an unstable second mode near the continuous spectrum point or near the synchronization point? We attempt to answer these questions in latter sections.

\section{Interaction of Acoustic Waves with the sharp-tipped wedge}

After the mean flow was computed, two-dimensional acoustic disturbances at different incidence angles of 0,10 , 20, and 30 degrees were introduced at the outer boundary of the computational domain and time-accurate simulations were performed. Computations were performed with the slow and fast acoustic waves at 0 degrees incidence angle and were performed with the slow wave for the other incidence angles. The amplitude of these forced acoustic disturbances was given a small value of $\tilde{p}_{a c} / p_{\infty}=1 * 10^{-5}$ to ensure that the disturbances evolving in the boundary layer remained in the linear regime. We present the results for a frequency of $F=0.95^{*} 10^{-4}(137 \mathrm{kHz})$. Figures $6(\mathrm{a}-\mathrm{h})$ show the unsteady density fluctuations at a fixed time for the acoustic incidence angle of $0,10,20$ 
and 30 degrees obtained with the slow acoustic wave and Figs. 6(i, j) show the results for the 0 deg with the fast acoustic wave. For each incidence angle, the top figure shows the density fluctuations for the entire domain and the bottom figure depicts the results inside the boundary layer. The observations are similar to our previous computational results ${ }^{20}$ for the flow over a flat plate at a supersonic Mach number of 4.5. The perturbation field can be divided into four regions. One region is the area outside the shock where the acoustic waves propagate uniformly. The second region is the shock layer across which the acoustic waves are transmitted. The third region is the area between the shock and the boundary layer. This region consists of the transmitted external acoustic field and the disturbances that are radiated from the boundary layer. The fourth region is the boundary layer where the boundary layer disturbances evolve. It is seen from Figs. 6(a-b) that at zero incidence angle for the slow mode, the acoustic waves are very weakly transmitted through the shock. It is also observed that the fluctuations inside the boundary layer are larger than the amplitude of the incoming acoustic field. It suggests that the boundary layers are perturbed strongly starting from the nose region. With increasing incidence angles Figs. 6(c-h), it is noted that the acoustic waves are still weakly transmitted through the shock in the lower part of the domain while they are strongly transmitted through the shock in the upper part as evident from the disturbed flow field between the wedge surface and the shock. Another observation is that the fluctuations inside the boundary layer in the lower part are lower than that in the upper part. Figures $6(i, j)$ display the density fluctuations induced by the fast mode at 0 degrees incidence angle. It is seen that the fast acoustic mode is transmitted strongly across the shock compared to the slow mode case, Figs. 6(a,b). The perturbations inside the boundary layer are larger than the amplitude of the incoming wave.

Figures 7(a-d) show the amplitude of the pressure fluctuations at the lower and upper side of the wall for the incident angles of $0,10,20$, and 30 degrees. Figure 7 also includes the results from the parabolized stability equations (PSE) computations obtained for the same mean boundary layer profiles. The growth of the disturbances agrees very well with the PSE results a short distance downstream of leading edge. Figure 7(a) shows the results for the slow and the fast modes at zero incidence angles. The figure for the slow wave clearly shows the initial generation and the eventual exponential growth of the instability waves inside the boundary layer. The slow acoustic wave whose wavelength $(5.29 \mathrm{~mm})$ is close to the wavelength $(5.16 \mathrm{~mm})$ of the slow mode (Mode S) near the leading edge transforms into instability waves rather directly. Similarly, the fast acoustic mode whose wavelength $(7.41 \mathrm{~mm})$ is close to the wavelength $(7.41 \mathrm{~mm})$ of the fast mode (Mode F) near the leading edge synchronizes with the instability mode near the leading edge region. As was observed in Fig. 5(a), the fast mode does not evolve into an unstable second mode as the Reynolds number increases. Figure 7(a) also illustrates that as the disturbance induced by the fast mode evolves downstream it first grows up to $x \sim 12 \mathrm{~cm}$. and then decreases to a smaller value near $x \sim 34 \mathrm{~cm}$. before it grows again due to the second mode instability. As we discussed earlier, the stable fast mode generated the unstable second mode near the continuous spectrum $(x=28 \mathrm{~cm}$.) or near the synchronization point $(x=40 \mathrm{~cm}$.). However, the unstable second mode generated by the fast mode is weak compared to that generated by the slow mode. The maximum amplitudes obtained in different cases are given in Table 4. The maximum amplitude reached in the slow acoustic wave case is about 0.00154 and it is about 0.00044 in the fast acoustic case. Hence, the slow mode is about four times more efficient in generating the unstable mode compared to the fast mode. From these values the receptivity coefficients defined by the initial amplitude of the pressure fluctuations at the wall non-dimensionalized by the free-stream acoustic pressure can be evaluated.

$$
C_{\text {recpt }, p_{\text {wall }}}=\frac{\left(p_{\text {wall }}\right)_{n}}{p_{a c}}
$$

The computed receptivity coefficient associated with the slow acoustic wave is about

$$
C_{\text {recpt, } p_{\text {wall }}, S}=2.5
$$


Table 4. Maximum amplitudes of pressure fluctuations for different incident angles.

\begin{tabular}{|c|c|c|c|}
\hline $\boldsymbol{\theta}_{\mathbf{y}}$ & Lower & Upper & $\begin{array}{c}\text { Ratio } \\
=\text { Upper/Lower }\end{array}$ \\
\hline $\begin{array}{c}0 \\
\text { Slow }\end{array}$ & 0.00154 & 0.00154 & 1.0 \\
\hline $\begin{array}{c}0 \\
\text { Fast }\end{array}$ & 0.00044 & 0.00044 & 1.0 \\
\hline $\begin{array}{c}10 \\
\text { Slow }\end{array}$ & 0.00093 & 0.00234 & 2.5 \\
\hline $\begin{array}{c}20 \\
\text { Slow }\end{array}$ & 0.00058 & 0.00270 & 4.6 \\
\hline $\begin{array}{c}30 \\
\text { Slow }\end{array}$ & 0.00038 & 0.00230 & 6.0 \\
\hline $\begin{array}{c}0 \\
\text { Vorticity }\end{array}$ & 0.00035 & 0.00035 & 1.0 \\
\hline
\end{tabular}

Figures 7(b-d) show the pressure fluctuations at the upper and the lower walls for different incidence angles 10, 20, and 30 degrees. Figures show that the maximum amplitudes are consistently smaller on the lower (windward) side compared to the upper (leeward) side. This effect increases with increasing angles of incidence. Table 4 gives the maximum amplitudes reached on the lower and the upper walls for the incidence angles 10, 20, and 30 degrees. The maximum amplitudes of the pressure fluctuations reached on the upper wall for the different incidence angles 0 , 10,20 , and 30 degrees are $0.0015,0.0023,0.0027$, and 0.0023 , respectively. The maximum amplitudes reached on the lower walls are $0.0015,0.00093,0.00058,0.00038$, respectively. Hence the receptivity becomes weaker on the lower side compared to the upper side with increasing incidence angles. The maximum amplitude on the upper surface increased by a factor of 1.8 for 20 degrees incidence angle and then decreases slowly for larger incidence angles.

To see how the wavenumber of the fluctuations inside the boundary layer varies along the streamwise direction and how they compare with the wavenumber obtained from the linear stability computations, the wavenumber of the pressure fluctuations along the wall is computed. This is achieved by first decomposing the fluctuations in to harmonic components of the form

$$
p(x, z, t)=\tilde{p}(x) e^{-i \omega t}+c \mathcal{C}
$$

and then evaluating the wavenumber of the fluctuations from the expression

$$
\begin{aligned}
\alpha(x) & =\left(\frac{1}{i \tilde{p}(x)} \frac{\partial \tilde{p}(x)}{\partial x}\right) \\
& =\alpha_{r}+i \alpha_{i}
\end{aligned}
$$

Figure 8 shows the pressure fluctuations along the surface at a fixed time in a linear scale. Figures $8(a, b)$ display the disturbances induced by the slow acoustic wave at 0 degrees incidence angles. Figure 8(a) depicts the results for the entire domain and Fig. 8(b) depicts the results near the leading edge region. Similarly, Figs. 8(c, d) show the results for the fast acoustic wave at 0 degrees incidence angles. Figures $8(\mathrm{e}, \mathrm{f})$ show the results for the 20 degree incidence angles acoustic wave on the bottom surface and Figs. $8(\mathrm{~g}, \mathrm{~h})$ show the results for the top surface. The first observation is that except for the disturbances induced by the fast acoustic wave, the wave pattern behaves smoothly for the entire domain without much modulation. In the case of the fast acoustic waves, the waves behave smoothly 
up to $x \sim 30 \mathrm{~cm}$., and between the region of $x \sim 30$ to $40 \mathrm{~cm}$., the disturbance amplitudes decrease to small values and the wave patterns consists of short and long wavelengths.

Figure 9 shows the computed wavenumber variation along the streamwise direction. Figure 9(a) gives the results for both the slow and fast acoustic waves cases while Fig. 9(b) gives the results for the 20 degrees incidence angle case. The figures also include the wavenumber distributions obtained from linear stability computations for the slow and the fast modes. Downstream of the leading-edge region $x>10.0 \mathrm{~mm}$, the wavenumber distributions computed using Eq. (23) agree very well with the linear stability theory results in all the cases. The wavenumber variations very near the nose region are shown in the inserts in Fig. 9(a). The wavenumber first decreases up to $x \sim 1 \mathrm{~mm}$, it then increases slowly for $x>1 \mathrm{~mm}$ and merges with the slow and the fast instability modes around $x \sim 10 \mathrm{~mm}$. The two solutions, from the simulation and the linear stability calculations, continue to agree beyond this point for the slow modes. The fast stability mode is also excited very near the leading edge as is evident by the agreement between the linear stability and the numerical simulation results. At a location close to $x \sim 200 \mathrm{~mm}$, the wavenumber starts to oscillate and merges with the values corresponding to the slow mode around $x \sim 400 \mathrm{~mm}$. This is the region where the energy is transferred to the unstable second mode from the fast mode. The locations corresponding to the continuous spectrum and to the synchronization point are also marked in the figure.

Figure 10 displays the amplitude variation of the density at several axial stations $x=50,200,350$, and $400 \mathrm{~mm}$ generated by the slow acoustic waves at 0 degrees incidence angles. The results are compared with the eigenfunctions obtained from the PSE calculations. As expected from the previous wavenumber and the amplitude comparisons, the simulation results agree with the PSE results starting from the leading edge region. This confirms that the slow instability mode is generated near the leading edge by the free stream slow acoustic wave. Similarly, Fig. 11 shows the amplitude distribution of the density generated by the fast acoustic waves at 0 degrees incidence angles. The eigenfunction distributions for the slow and fast instability modes are also included for comparison. As observed in Fig. 6(i), the fast acoustic waves transmit through the shock and perturb the region above the boundary layer. This is evident in Figs. (11) by the appearance of disturbances outside the boundary layer. At the stations $x=$ 200 and $250 \mathrm{~mm}$, the eigenfunctions for the fast instability mode agree with that obtained from the simulation. This confirms that the fast instability mode is generated near the leading edge by the free stream fast acoustic wave. Beyond $x>350 \mathrm{~mm}$, the simulation results agree with the eigenfunctions for the slow instability mode. Hence the switching or the transformation from the fast instability mode to the unstable second mode should have occurred between the stations $x=275$ to $350 \mathrm{~mm}$. This observation favors the theory of Ref. [17] that the inter-modal energy exchange in this region causes the fast mode to switch to the unstable second mode.

\section{Interaction of Vorticity Waves with the sharp-tipped wedge}

Figures 12(a-d) show the density and the vertical velocity, $v$, perturbations generated by the interaction of vortical disturbances with the boundary layer. Figures 12(a), (b) display the density fluctuations near the leading edge and inside the boundary layer in the middle of the domain, respectively. Figures 12(c) and (d) similarly depict the fluctuations for the vertical velocity component. Since vortical disturbances do not contain density or pressure fluctuations in the free stream, density fluctuations appear only between the shock and the wall due to the interaction of vortical disturbances with the shock. The flow field revealed by the velocity perturbations can be divided, as discussed in the previous section, into four regions. One is the region outside the shock where the perturbations are uniform and governed by the linerized Euler equations. The second is the region between the shock and the boundary layer away from the leading edge. Here, the incoming vortical wave is diffracted towards the normal to the shock and the amplitude of the wave is increased across the shock. However, the diffracted waves do not penetrate the boundary layer. The third is the region between the shock and the wall near the leading edge. Here, the incoming vortical disturbances are diffracted and elongated and the amplitude is decreased across the shock. The fourth is the boundary layer region. Figures 12(a) and (d) show that the perturbations inside the boundary layer are generated starting from the leading edge.

Figures 13(a) and (b) depict the wall pressure fluctuations generated by the interaction of vortical disturbances with the wedge. Figure 13(a) displays the results in the linear scale, while Fig. 13(b) shows the results in the log scale. The results show that up to $x \sim 28 \mathrm{~cm}$, the oscillations consist of short wavelength disturbances that are modulated by long wavelength disturbances. Beyond $x \sim 28 \mathrm{~cm}$, the short wavelength disturbances merge into the unstable second mode wave. Figure 14 compares the wall pressure oscillations generated by the slow and fast acoustic wave and by the vortical disturbance. The maximum amplitudes obtained in different cases are given in Table 4. The maximum amplitudes generated by the interaction with these waves are $0.0015,0.00044$ and 0.00035 , respectively. Hence the fast acoustic wave is about 3.4 times less efficient than the slow acoustic wave, and the vorticity wave is about 4.2 times less efficient in generating the unstable second mode in an adiabatic boundary layer over a wedge. These numbers may change with frequencies, however, the conclusions will remain the same.

12

American Institute of Aeronautics and Astronautics 
$49^{\text {th }}$ AIAA Aerosciences Meeting, January 4-7, 2011, Orlando, Florida

Figure 15 shows the calculated wavenumber variation for this case. The wavenumbers computed from the linear instability are also included in the figure for comparison. It is interesting to see that the wavenumber of the fluctuations follows the wavenumber for the fast instability mode up to $x \sim 250 \mathrm{~mm}$ and jumps to the wavenumber for the slow instability mode near $x \sim 280 \mathrm{~mm}$. This is the location where the fast mode is absorbed into the continuous spectrum. Hence the switching or the transformation from the fast instability mode to the unstable second mode in this case occurs near the continuous spectrum.

Figures 16(a-i) show the amplitude distribution of the density at several streamwise locations $x=200$ to 425 $\mathrm{mm}$. The eigenfunction distributions for the slow and fast instability modes are also included in these figures for comparison. To understand the evolution of the waves along the streamwise direction, it is better to begin from the downstream locations and to trace backwards. Figure 16(i) displays the amplitude distribution at $x=425 \mathrm{~mm}$. The amplitude distribution from the simulation exhibit two peaks, one near $y=2.6 \mathrm{~mm}$ and the other near $y=2.2 \mathrm{~mm}$. The eigenfunction for the slow instability mode agrees with the simulation results up to the lower peak. The upper peak in the simulation results is the forced disturbance caused by the transmitted disturbance field. If we trace the peaks upstream, the lower peak is clearly seen at $x=400,350$ and $275 \mathrm{~mm}$. The lower peak is not discernable at $x=$ 375,325 and $300 \mathrm{~mm}$. However, the eigenfuntion for the slow instability mode agrees better with the simulation results from $x=275$ to $425 \mathrm{~mm}$.

\section{B. $7^{\circ}$ Half-Angle Sharp-Tipped Cone}

\section{Mean Flow}

Figure 17(a) and (b) show the computed mean density contours for the sharp-tipped cone. The flow field for the entire computational domain is shown in Fig. 17(a), while Fig. 17(b) shows a close-up view of the nose region. The mean flow boundary layer profiles and linear stability results were given in Ref. 24 . The boundary layer profiles slowly approach the Blasius similarity profile by $x=3.0 \mathrm{~cm}$. The maximum $\mathrm{N}$-factor obtained is about 7.0 and the corresponding frequency is $F=1.5^{*} 10^{-4}(217 \mathrm{kHz})$.

Similar to the wedge case, Figs. 18(a) and 18(b) show the evolution of the eigenvalues along axial direction for the slow (Mode S) and fast (Mode F) modes for the frequency $F=1.5^{*} 10^{-4}$. Figure 18(a) shows the evolution of phase speed $c_{r}$ and the growth rate $-\alpha_{i}$ and Fig. 18(b) shows the wavenumber $\alpha_{r}$ and the wavelength. The observations are the same as for the wedge case. As the fast mode evolves downstream, it merges with the continuous spectrum with phase speed $c_{r}=1.0$. For this frequency of $F=1.5 * 10^{-4}$ the merging occurs around $x=$ $281 \mathrm{~mm}$. Downstream of the continuous spectrum a new mode with slightly different eigenvalues and eigenfunctions emerges. These points are also marked as red dots in Fig. 18(a). As the slow mode and the new fast mode evolve downstream, the slow mode becomes the unstable Mack's second mode and the fast mode becomes the stable mode. The synchronization of the phase speeds for the slow and the fast modes occurs near $x=360 \mathrm{~mm}$. The growth rates of the slow mode and the fast mode at this point are $-\alpha_{i}=0.005$ and -0.02 , respectively. The continuous spectrum and the synchronization point are also marked as vertical lines in Figs 18(a) and (b), respectfully.

\section{Interaction of Slow and Fast Acoustic Waves with the cone}

As in the previous case, after the mean flow was computed, two-dimensional slow and fast acoustic disturbances (with an amplitude of $\tilde{p}_{a c} / p_{\infty}=1 * 10^{-5}$ ) at an incidence angle of 0 degrees were introduced at the outer boundary of the computational domain and time-accurate simulations were performed. Here, we present the results for a frequency of $F=1.5^{*} 10^{-4}(217 \mathrm{kHz})$. Contours of the density fluctuations generated by the slow acoustic waves are shown in Fig. 19(a) for the nose region, and in Fig. 19(b) for the tip region. Figure 19(c) displays the contours of the density fluctuations generated by the fast acoustic wave for the entire domain and Fig. 19(d) displays the results in the middle of the domain from $x=12$ to $22 \mathrm{~cm}$. The flow features are similar to the wedge case. For the slow mode, the acoustic waves are very weakly transmitted through the shock. It is also observed that the fluctuations inside the boundary layer are larger than the amplitude of the incoming acoustic field. It suggests that the boundary layer is perturbed strongly starting from the nose region. The fast acoustic mode is transmitted strongly across the shock as compared to the slow mode case. The perturbations inside the boundary layer are larger than the amplitude of the incoming wave. Fig. 19(d) also shows that the amplitude inside the boundary layer does not increase or decrease uniformly. In some regions it increases and in some region it decreases to very small values.

Figures 20(a-b) show the amplitude of the pressure fluctuations along the surface of the cone for the slow and the fast acoustic waves. Figure 20 also includes the results from the parabolized stability equations (PSE) computations obtained for the same mean boundary layer profiles. The figure for the slow wave clearly shows the initial generation and the eventual exponential growth of the instability waves inside the boundary layer. The slow acoustic wave whose wavelength $(3.35 \mathrm{~mm}$ ) is close to the wavelength of the slow mode (Mode S) near the leading edge 
$(3.47 \mathrm{~mm})$ transforms into instability waves rather directly. Similarly, the fast acoustic mode whose wavelength $(4.69 \mathrm{~mm})$ is close to the wavelength of the fast mode (Mode F) near the leading edge $(4.64 \mathrm{~mm})$ synchronizes with the instability mode near the leading edge region. As was observed in Fig. 18(a), the fast mode does not evolve into an unstable second mode as the Reynolds number increases. Figure 20(b) shows that as the disturbance induced by the fast mode evolves downstream it first grows up to $x \sim 12 \mathrm{~cm}$, then decreases and oscillates before it grows due to the second mode instability. The unstable second mode generated by the fast mode is weak compared to that generated by the slow mode. The maximum amplitude reached in the slow acoustic wave case is about 0.213 and it is about 0.0023 in the fast acoustic case. Hence, the slow mode is about ninety times more efficient in generating the unstable mode compared to the slow mode. From these values the receptivity coefficients defined by the initial amplitude of the pressure fluctuations at the wall non-dimensionalized by the free-stream acoustic pressure can be evaluated.

$$
C_{\text {recpt }, p_{\text {wall }}}=\frac{\left(p_{\text {wall }}\right)_{n}}{p_{a c}}
$$

The computed receptivity coefficients associated with the slow acoustic wave is about

$$
C_{\text {recpt, } p_{\text {wall }}, S}=5.0
$$

Figure 21 shows the computed wavenumber variation along the streamwise direction for the slow and fast acoustic waves cases. The figures also include the wavenumber distributions obtained from linear stability computations for the slow and the fast instability modes. Downstream of the leading-edge region $x>10.0 \mathrm{~mm}$, the wavenumber distributions computed using Eq. (23) agree very well with the linear stability theory results for the both cases. The wavenumber variations very near the nose region are shown in the inserts in Fig. 21. The wavenumber first decrease up to $x \sim 1.5 \mathrm{~mm}$, it then increases slowly for $x>1.5 \mathrm{~mm}$ and merges with the slow and the fast instability modes around $x \sim 10 \mathrm{~mm}$. The two solutions, from the simulation and the linear stability calculations, continue to agree beyond this point for the slow modes. The fast stability mode is also excited very near the leading edge as is evident by the agreement between the linear stability and the numerical simulation results. At a location close to $x=120 \mathrm{~mm}$, the wavenumber starts to oscillate and shifts to a lower value of 0.13 . Further downstream around $x \sim 360 \mathrm{~mm}$, the wavenumber jumps to the value corresponds to the slow wave. The synchronization point for this case also occurs near $x \sim 360 \mathrm{~mm}$. Hence, the energy is transferred from the fast mode to the unstable second mode near the synchronization point.

\section{Interaction of Axisymmetric Slow Acoustic Waves at Incidence with the cone}

Figures 22 and 23 show the results for the interaction of an axisymmeric slow acoustic wave with the cone. The axisymmetric acoustic waves are governed by the zeroth order Bessel function. The simulations are performed for a frequency of $F=1.5^{*} 10^{-4}$ and an incidence wavenumber of $\alpha_{\mathrm{n}}=0.05$. The acoustic field oscillates and decays in the radial direction. Figure 22 presents the density perturbations near the nose region (Fig. 22(a)) and near the tip region (Fig. 22(b)). It is seen as we mentioned previously, that the acoustic field oscillates and decays in the free stream. The acoustic field is concentrated along the axis of the cone. This resembles the acoustic field that will appear in wind tunnels with axisymmetric nozzles. As in the interaction of plane acoustic waves, the disturbances appear inside the boundary layer very near the tip of the cone. Figure 23 displays the pressure fluctuations along the wall. The evolution is similar to the previous case. The maximum amplitudes generated by the acoustic disturbances are 0.213 and 0.267 for the plane and the circular waves respectively. Hence, the axisymmetric wave with the wave number in the radial direction of $\alpha_{n}=0.05$ is about 1.25 times more efficient than the plane acoustic wave. As in the previous cases, Fig. 24 shows the wavenumber variation along the streamwise direction for this case. The wavenumber initially oscillates between 0.23 and 0.15 and further downstream close to $x \sim 30 \mathrm{~mm}$ merges with the wavenumber for the slow instability mode.

\section{Interaction of Vorticity Waves with the sharp-tipped cone}

Figures 25(a-d) show the contours of the density and the axial velocity, $u$, perturbations generated by the interaction of vortical disturbances with the boundary layer. Figures 25 (a) and (b) display the density fluctuations for the entire domain and in the middle of the domain $x=30$ to $35 \mathrm{~cm}$, respectively. Figures 25 (c) and (d) similarly depict the fluctuations for the axial velocity component. Since vortical disturbances do not contain density or pressure fluctuations in the free stream, density fluctuations appear only between the shock and the wall due to the interaction of vortical disturbances with the shock. The flow field revealed by the velocity perturbations are similar to that observed in the wedge case. 
Figures 26(a) and (b) depict the wall pressure fluctuations generated by the interaction of vortical disturbances with the cone. Figure 26(a) displays the results in the linear scale, while Fig. 26(b) shows the results in the log scale. The results show that the disturbances grow up to $x \sim 30 \mathrm{~cm}$, then decrease to a minimum value close to $x=33 \mathrm{~cm}$. and increase downstream due to the second mode instability. The maximum amplitude obtained in this case is about 0.078 . Table 5 summarizes the maximum amplitudes reached by the disturbances in different cases. The fast mode is about 93 times less efficient than the slow mode, the axi-symmetric mode at an incidence wavenumber of $\alpha_{n}=0.05$ is about 1.25 times more efficient and the vortical disturbance is about 2.7 times less efficient.

Table 5. Maximum amplitudes of pressure fluctuations for different cases $F=1.5 * 10^{-4}$.

\begin{tabular}{|c|c|c|}
\hline $\boldsymbol{\theta}_{\mathbf{y}}$ & $\begin{array}{c}\text { Max. } \\
\text { amplitude }\end{array}$ & $\begin{array}{c}\text { Ratio } \\
=\mathbf{m a x} / \mathbf{m a x}_{\text {slow }}\end{array}$ \\
\hline $\begin{array}{c}0 \\
\text { Slow }\end{array}$ & 0.213 & 1.0 \\
\hline $\begin{array}{c}0 \\
\text { Fast }\end{array}$ & 0.0023 & $1 / 93$ \\
\hline $\begin{array}{c}\alpha_{\mathrm{n}}=0.05 \\
\text { Axi_sym. }\end{array}$ & 0.267 & 1.25 \\
\hline $\begin{array}{c}0 \\
\text { Vorticity }\end{array}$ & 0.078 & $1 / 2.7$ \\
\hline
\end{tabular}

Figure 27 shows the calculated wavenumber variation for this case. The wavenumbers computed from the linear instability are also included in the figure for comparison. The wave number oscillates around 0.155 until $x \sim 300$ $\mathrm{mm}$, then increases slowly and merges with the wavenumber for the slow mode instability. It is observed that the wavenumber in the freestream for the vorticity wave is also about 0.153 (Table 2). This suggests that the disturbances in the boundary layer up to $x \sim 300 \mathrm{~mm}$ are dominated by the transmitted vorticity waves. It is also noticed that the large oscillations in the wavenumber and the minimum amplitude in the pressure (Fig. 26(a)) occur near the same location around $x \sim 330 \mathrm{~mm}$. At this point, we do not know the significance of this coincidence, however, it may be a hint in identifying the mechanism for generating the unstable second mode in this case. It would be interesting to compare the shape of the amplitude distributions with the eigenfunctions for the slow and the fast modes. That will be studied in our future work.

\section{Conclusions}

The receptivity of the instability waves to two-dimensional acoustic and vortical waves in hypersonic flows over a sharp-tipped wedge and a cone at a free stream Mach number of 6 and at a Reynolds number of $10.4 * 10^{6} / \mathrm{meter}$ was numerically investigated. The effect of the incidence angles of the acoustic waves was also investigated. Both the steady and unsteady solutions were obtained by solving compressible Navier-Stokes equations using the $5^{\text {th }}$ order accurate weighted essentially non-oscillatory (WENO) scheme for space discretization and using a third-order total-variation-diminishing (TVD) Runge-Kutta scheme for time integration.

The results show that the slow and the fast acoustic waves directly synchronize with the slow and fast instability modes very close to the leading edge. For the wedge case, the receptivity coefficient for the slow mode based on the wall pressure fluctuations near the leading edge is about 2.5 times the amplitude of the freestream slow acoustic wave. We also found that the amplitude of the instability waves generated by the slow acoustic waves is about three times larger than that for the case of fast acoustic waves. This factor is smaller than other findings ${ }^{16,20}$ because of the frequency we selected in this computation reached an N-Factor of only 4 . The general conclusion agrees with other findings that forcing by slow acoustic wave is much more relevant in the transition process involved in supersonic and hypersonic boundary layers with adiabatic wall conditions. The results also showed that the receptivity on the leeward side increases slightly with increasing incidence angle and decreases on the windward side. The receptivity is the highest, by a factor of 1.8, for an incidence angle of 20 degrees. The vortical disturbances also generate the second mode disturbances in hypersonic boundary layers. The receptivity coefficient is about four 
times smaller than that for the slow acoustic wave. The wavenumber of the fluctuations showed that the vorticity waves generate the fast mode near the leading edge and the switching or the transformation of the fast instability mode to the slow instability mode in this case occurs near the continuous spectrum.

In the cone case, the receptivity coefficient for the slow mode is about 5 times the amplitude of the freestream slow acoustic wave. It is also found that the amplitude of the instability waves generated by the slow acoustic waves is about ninety times larger than that for the case of fast acoustic waves. This also illustrates the importance of the slow acoustic wave compared to the fast wave in causing transition in adiabatic hypersonic boundary layers. The receptivity to axisymmetric acoustic waves is slightly higher than that due to plane acoustic waves. The receptivity coefficient is about 1.25 times that for the plane acoustic wave. Axisymmetric vortical disturbances also generate unstable second modes in hypersonic boundary layers. The receptivity coefficient is about three times smaller than that for the slow acoustic wave. We have not compared the shape of the amplitude distributions with the eigenfunctions for the slow and the fast modes in this paper. That will be studied in our future work.

\section{References}

${ }^{1}$ Mack, L., “Boundary Layer Stability Theory,” JPL Report No. 900-277 Rev. A, Pasadena, Calif, Nov. 1969.

${ }^{2}$ Kendall,J. M., "Wind Tunnel Experiments Relating to Supersonic and Hypersonic Boundary-Layer Transition ," AIAA J., Vol. 13, No. 3, 1975, pp. 290-299.

${ }^{3}$ Stetson, K. F., Thompson, E. R., Donaldson, J. C., and Siler, L. G., "Laminar Boundary Layer Stability Experiments on a Cone at Mach 8, Part 1: Sharp Cone," AIAA $22^{\text {nd }}$ Aerospace Sciences Meeting, Reno, Nevada, January 9-12, AIAA Paper 83$1761,1983$.

${ }^{4}$ Stetson, K. F., and Kimmel, R. L., “On Hypersonic Boundary-Layer Stability,” AIAA Paper 92-0737, January 1992.

${ }^{5}$ Blanchard, A.E., “An Investigation of Wall-Cooling Effects on Hypersonic Boundary-Layer Stability in a Quiet Wind Tunnel”, Ph.D. Dissertation, Old Dominion University, Dept. of Mechanical Engineering, Norfolk, VA, December, 1995.

${ }^{6}$ Lachowicz, J, T., Chokani, N., and Wilkinson, S.P., "Boundary-Layer Stability Measurements in a Hypersonic Quiet Tunnel”, AIAA Journal, Vol. 34, No. 12, December 1996.

${ }^{7}$ Wilkinson, S. P., "A Review of Hypersonic Boundary Layer Stability Experiments in a Quiet Mach 6 Wind Tunnel”, AIAA Paper 97-1819, June 1997.

${ }^{8}$ Maslov, A. A., Shiplyuk, A. N., Sidorenko, A. A and Arnal, D., "Leading Edge Receptivity of a Hypersonic Boundary layer on a Flat Plate," J. Fluid Mech., Vol. 426, 2001, pp. 73-94.

${ }^{9}$ Wheaton, B., Juliano, T.J., Berridge, D.C., Chou, A., Gilbert, P.L., Casper, K.M., Steen, L.E., Schneider, S., and Johnson, H.B., "Instability and Transition Measurements in the Mach 6 Quiet Tunnel," AIAA Paper 2009-3559, June 2009.

${ }^{10}$ Casper, K.M., Beresh, S.J., Henfling, J.F., Spillers, R.W., Pruett, B., and Schneider, S.P., "Hypersonic Wind-Tunnel Measurements of Boundary-Layer Pressure Fluctuations,” AIAA Paper 2009-4054, Jun 2009.

${ }^{11}$ Malik, M. R., "Boundary-Layer Transition Prediction Toolkit," AIAA Paper 1997-1904.

${ }^{12}$ Gushin, V. R., and Fedorov, A. V., "Excitation and development of unstable disturbances in supersonic boundary layer," Fluid Dynamics, Vol. 25, No. 3, 1990, pp. 344-352.

${ }^{13}$ Fedorov, A. V., and Khokhlov, A. P., "Prehistory of Instability in a Hypersonic Boundary Layer," Theoret. Comput. Fluid Dynamics, Vol. 14, pp. 359-375, 2001.

${ }^{14}$ Fedorov, A. V., and Khokhlov, A. P., “ Excitation of Unstable Modes in a Supersonic Boundary Layer by Acoustic Waves," Fluid Dynamics, Vol. 26, No. 4, pp. 531-537, July 1991.

${ }^{15}$ Fedorov, A. V., and Khokhlov, A. P., "Sensitivity of a Supersonic Boundary Layer to Acoustic Disturbances," Fluid Dynamics, No. 27, pp. 29-34, 1992

${ }^{16}$ Fedorov, A.V., "Receptivity of a High-Speed Boundary Layer to Acoustic Disturbances," Journal of Fluid Mechanics, Vol. 491, 2003, pp. 101-129.

${ }^{18} \mathrm{Ma}$, Y. and Zhong, X., "Receptivity of a Supersonic Boundary Layer over a Flat Plate. Part 1. Wave Structures and Interactions," Journal of Fluid Mechanics, Vol. 488, 2003, pp. 31-78.

${ }^{19}$ Ma, Y. and Zhong, X., "Receptivity of a Supersonic Boundary Layer over a Flat Plate. Part 2. Receptivity to Freestream Sound," Journal of Fluid Mechanics, Vol. 488, 2003, pp. 79-121.

${ }^{20}$ Malik, M. R., and Balakumar, P., “Acoustic receptivity of Mach 4.5 boundary layer with leading edge bluntness,” Theor. Comput. Fluid Dyn. (2007) 21:323-342.

${ }^{21}$ Kara, K., Balakumar, P., and Kandil, O.A., "Receptivity of Hypersonic Boundary Layers Due to Acoustic Disturbances over Blunt Cone," AIAA Paper 2007-945, Jan. 2007.

${ }^{22}$ Kara, K., Balakumar, P., and Kandil, O.A., "Effects of Nose Bluntness on Stability of Hypersonic Boundary Layers over a Blunt Cone," AIAA Paper 2007-4492, June 2007.

${ }^{23}$ Kara, K., Balakumar, P., and Kandil, O.A., "Effects of Wall Cooling on Hypersonic Boundary Layer Receptivity over a Cone," AIAA Paper 2008-3734, June 2008.

${ }^{24}$ Balakumar, P., and Kegerise, M. A., "Receptivity of Hypersonic Boundary Layers over Straight and Flared Cones," AIAA Paper 2010-1545, January 2010.

${ }^{25}$ Shu, C.W., Essentially Non-Oscillatory And Weighted Essentially Non-Oscillatory Schemes For Hyperbolic Conservation Laws, NASA/CR-97-206253 and ICASE Report No. 97-6. 
$49^{\text {th }}$ AIAA Aerosciences Meeting, January 4-7, 2011, Orlando, Florida

${ }^{26}$ Atkins, H.L., "High-Order ENO Methods for the Unsteady Compressible Navier-Stokes Equations," AIAA Paper 91-1557, 1991.

${ }^{27}$ Balakumar, P., Zhao, H., and Atkins, H., "Stability of Hypersonic Boundary Layers Over a Compression Corner," AIAA Paper 2002-2848, June 2002. 


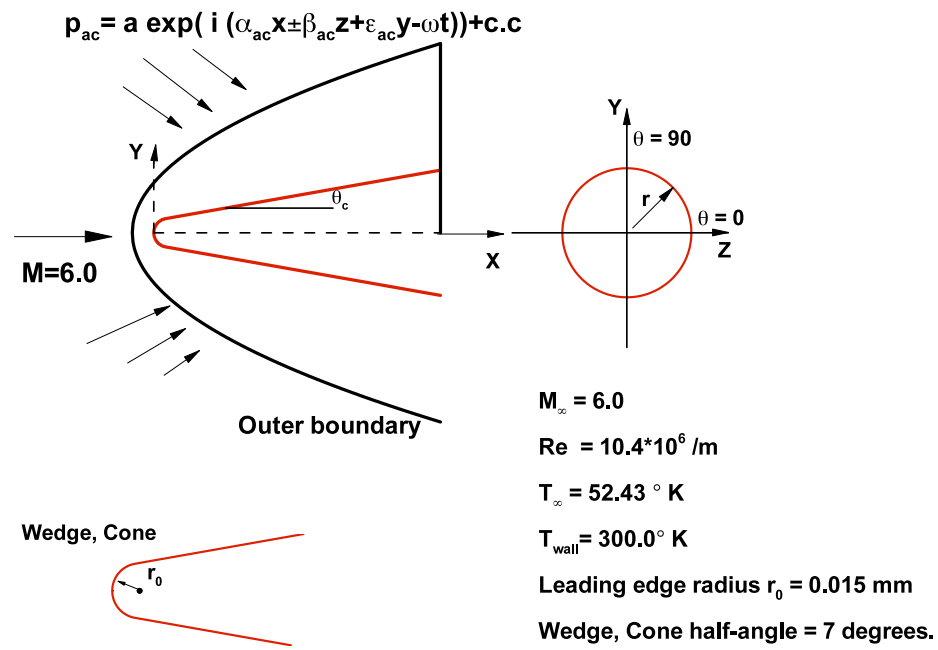

Figure 1. Schematic diagram of the computational model for flow over a 7-degree sharp-tipped wedge and a cone.

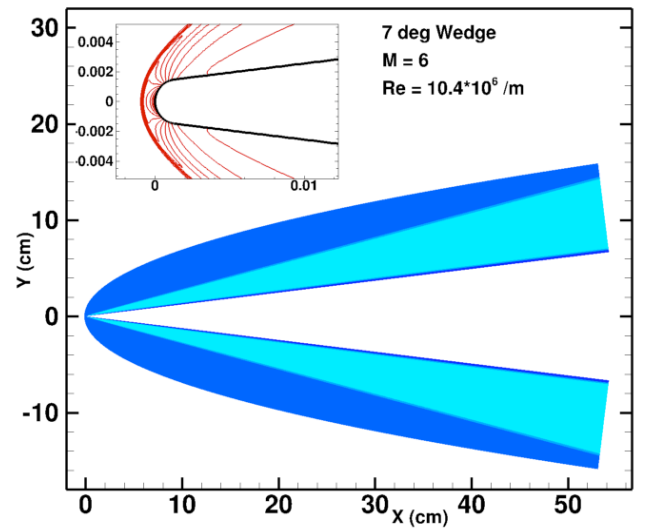

Figure 2. Mean density contours for flow over a 7-degree sharp-tipped wedge at Mach 6.

(a)

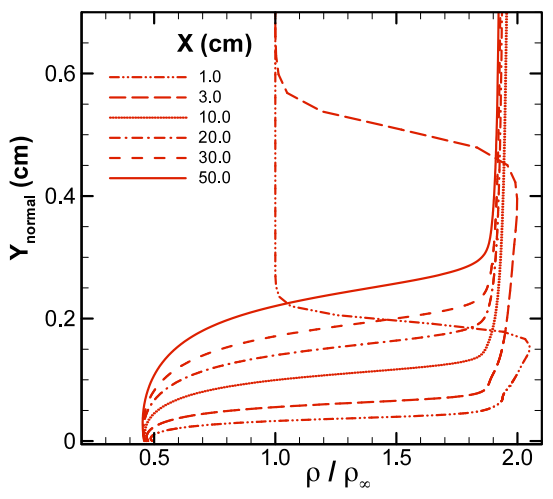

(b)

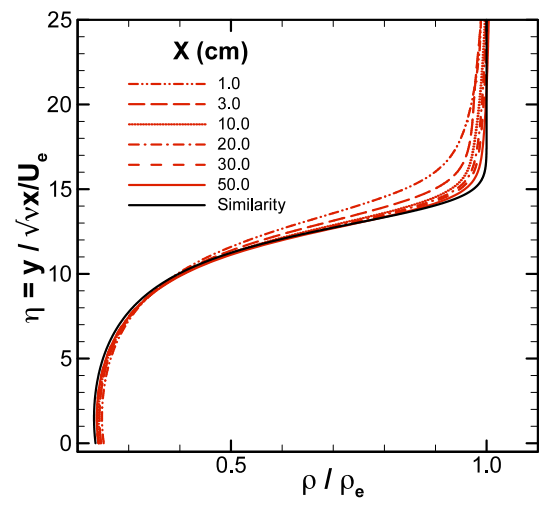

Figure 3. Boundary layer density profiles (a) in physical and (b) similarity coordinates at different axial locations for flow over a 7-degree sharp-tipped wedge at Mach 6. 

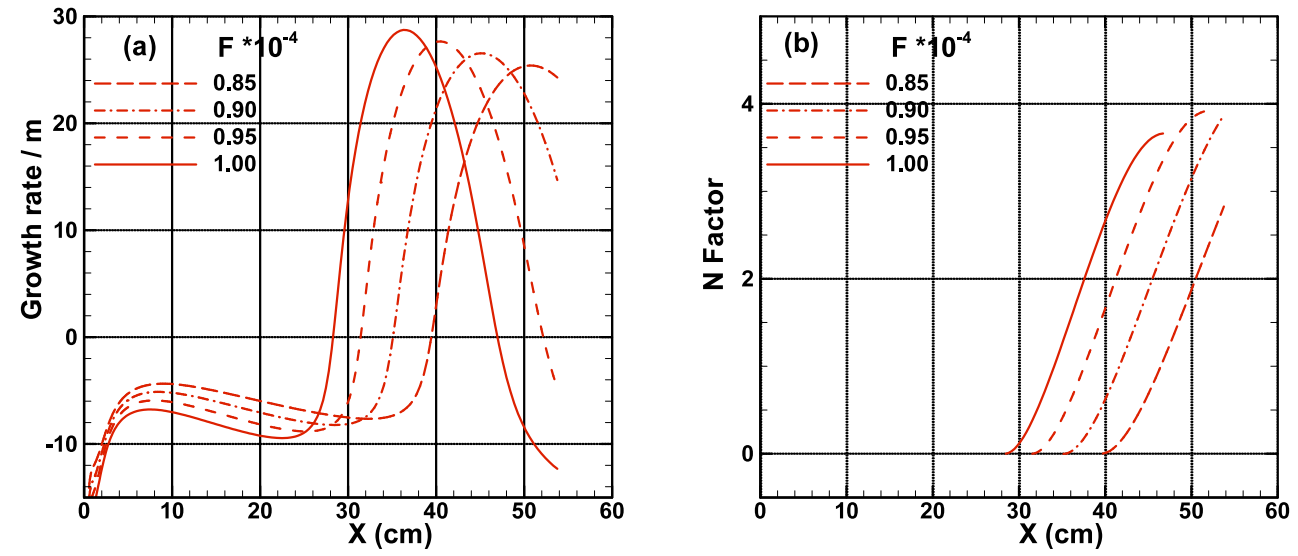

Figure 4. (a) Growth rate and (b) N-Factor curves computed from the linear stability analysis.

(a)

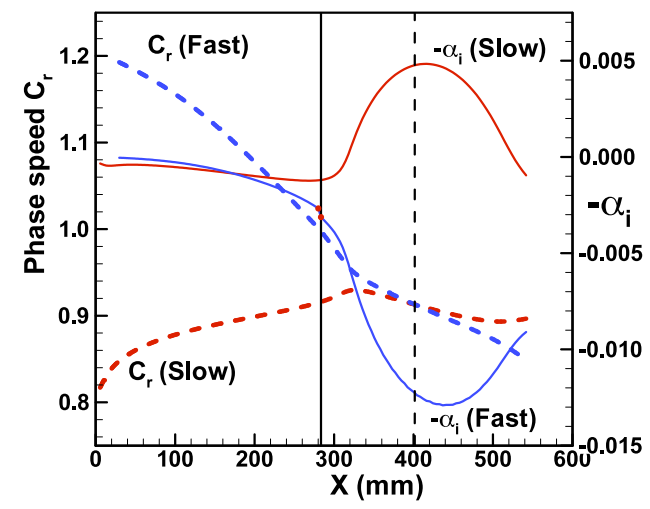

(b)

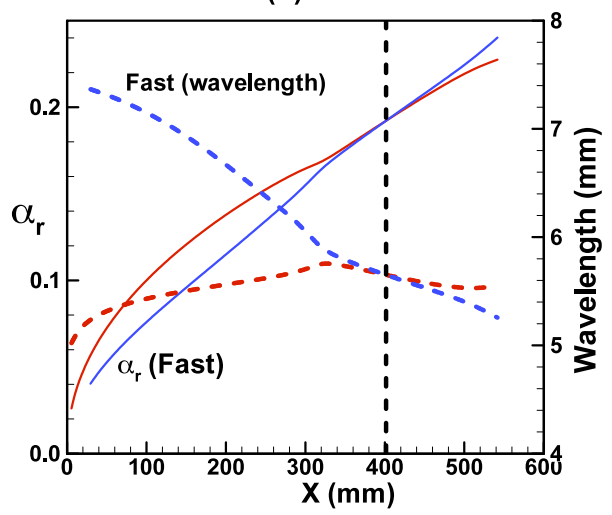

Figure 5. Eigenvalues from the linear stability computations for fast (blue) and slow (red) modes for the frequency $F=0.95 * 10^{-4}$. (a) Phase speed $c_{r}$ and growth rates $-\alpha_{i}$ and (b) wavenumber $\alpha_{r}$ and wavelength. 
$49^{\text {th }}$ AIAA Aerosciences Meeting, January 4-7, 2011, Orlando, Florida
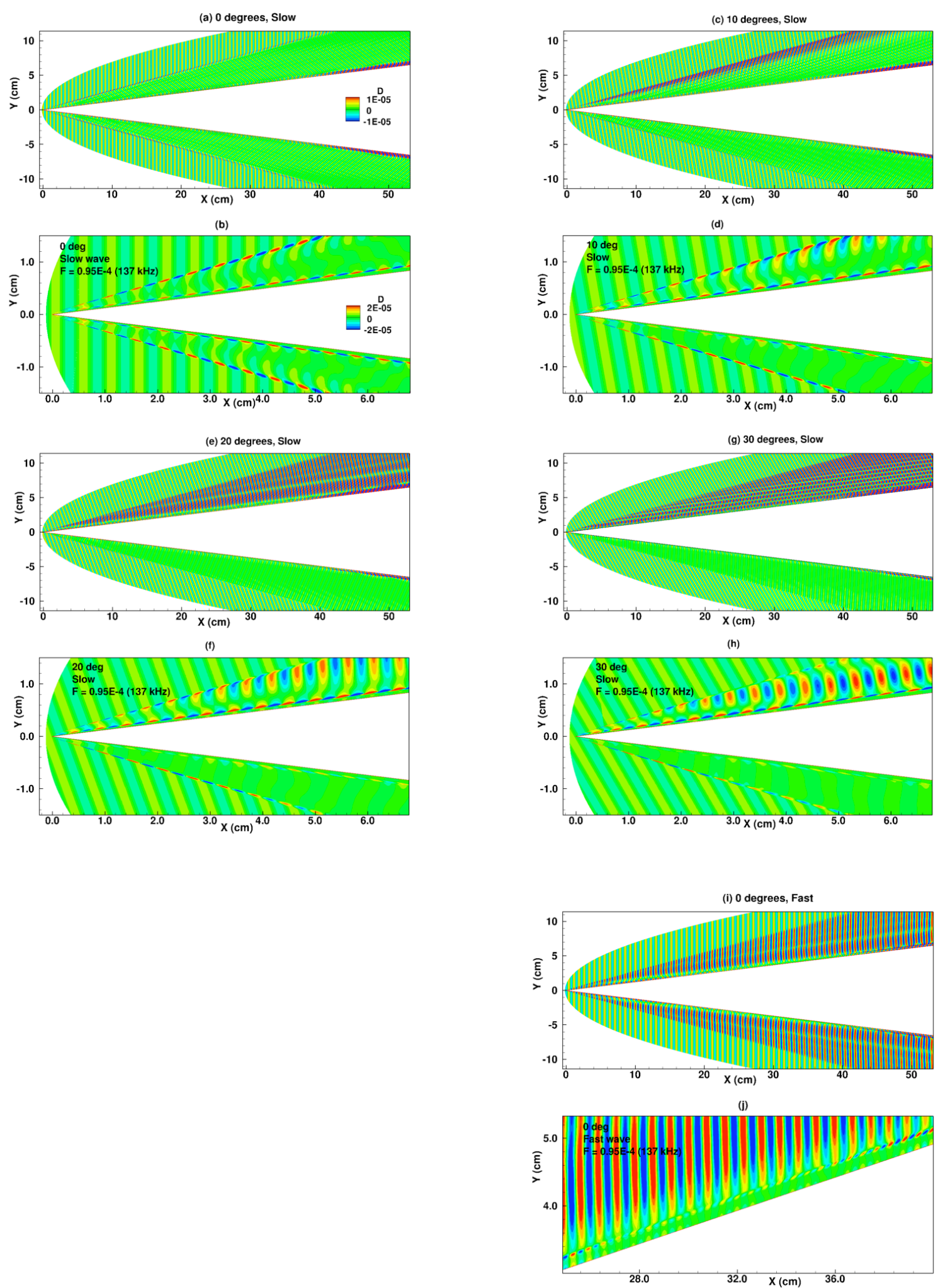

Figure 6. Density fluctuations generated by the interaction of a two-dimensional acoustic wave with the sharp-tipped wedge, incident angles $0,10,20,30$ degrees. $F=0.95 * 10^{-4}$. 
$49^{\text {th }}$ AIAA Aerosciences Meeting, January 4-7, 2011, Orlando, Florida
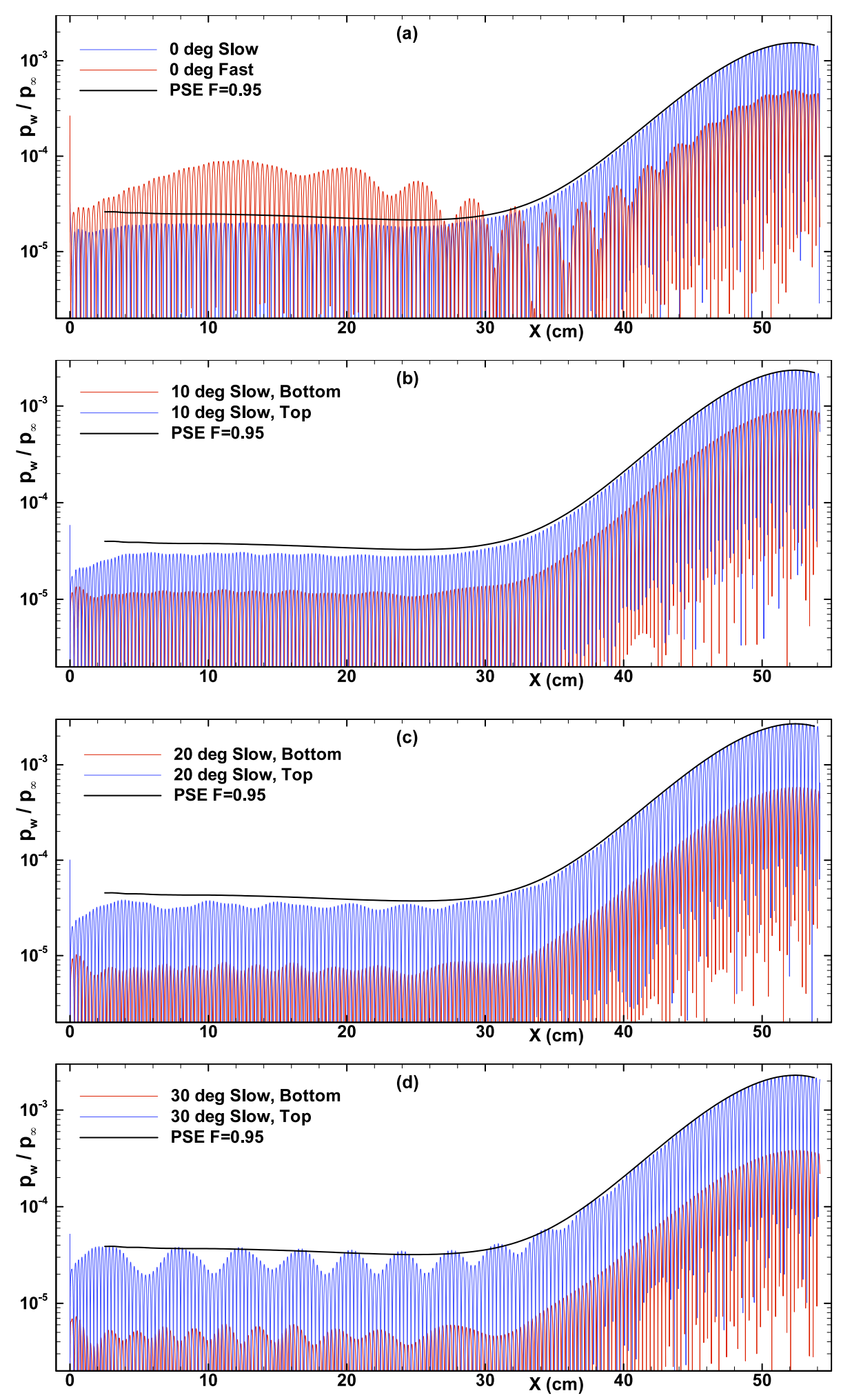

Figure 7. Pressure fluctuations at the top and bottom surfaces of the sharp-tipped wedge generated by twodimensional slow and fast acoustic waves at incident angles of $0,10,20$, and 30 degrees. $F=0.95 * 10^{-4}$. 
$49^{\text {th }}$ AIAA Aerosciences Meeting, January 4-7, 2011, Orlando, Florida
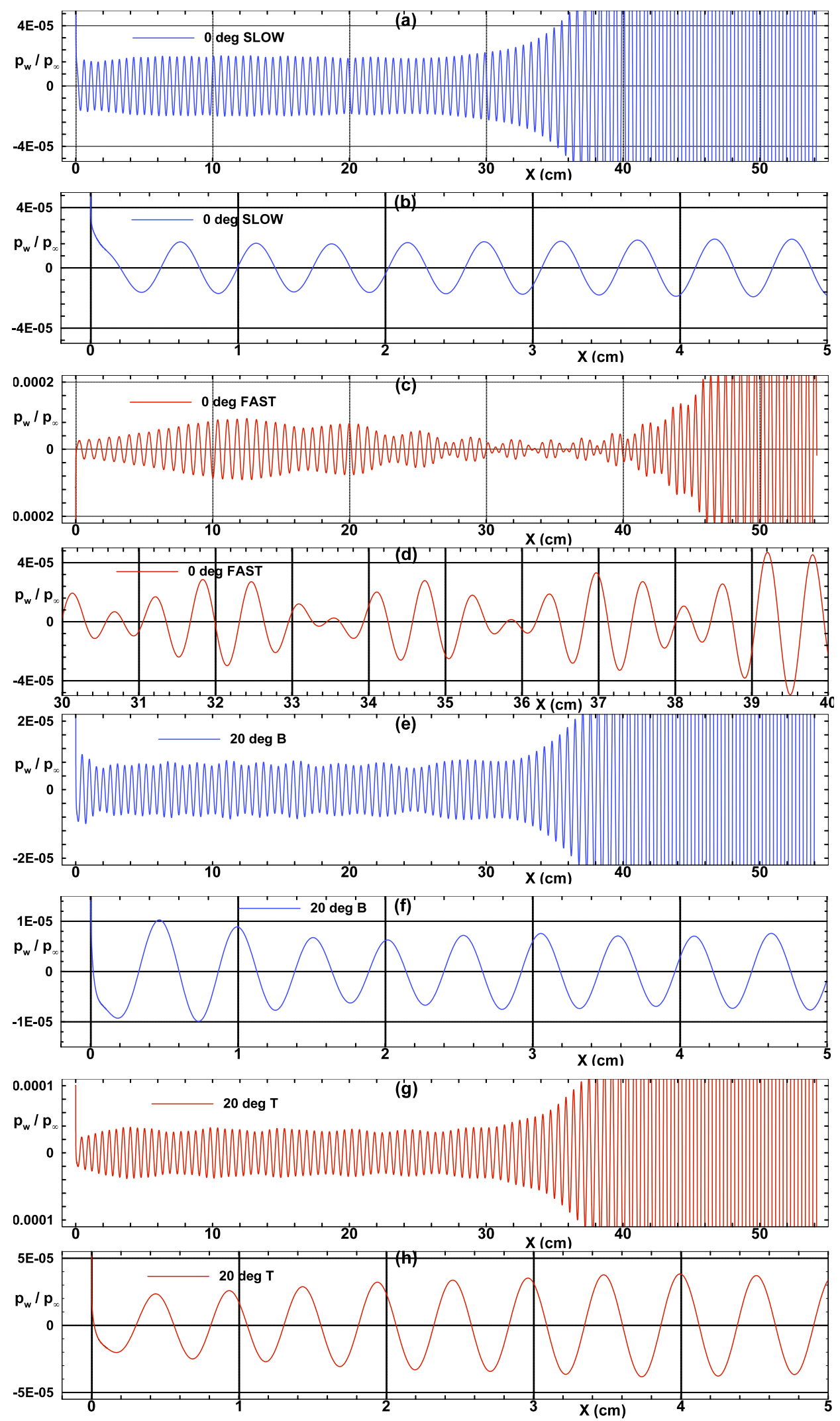

Figure 8. Pressure fluctuations along the surface of the sharp-tipped wedge generated by two-dimensional acoustic waves at incident angles of 0 and 20 degrees. $F=0.95 * 10^{-4}$.

American Institute of Aeronautics and Astronautics 

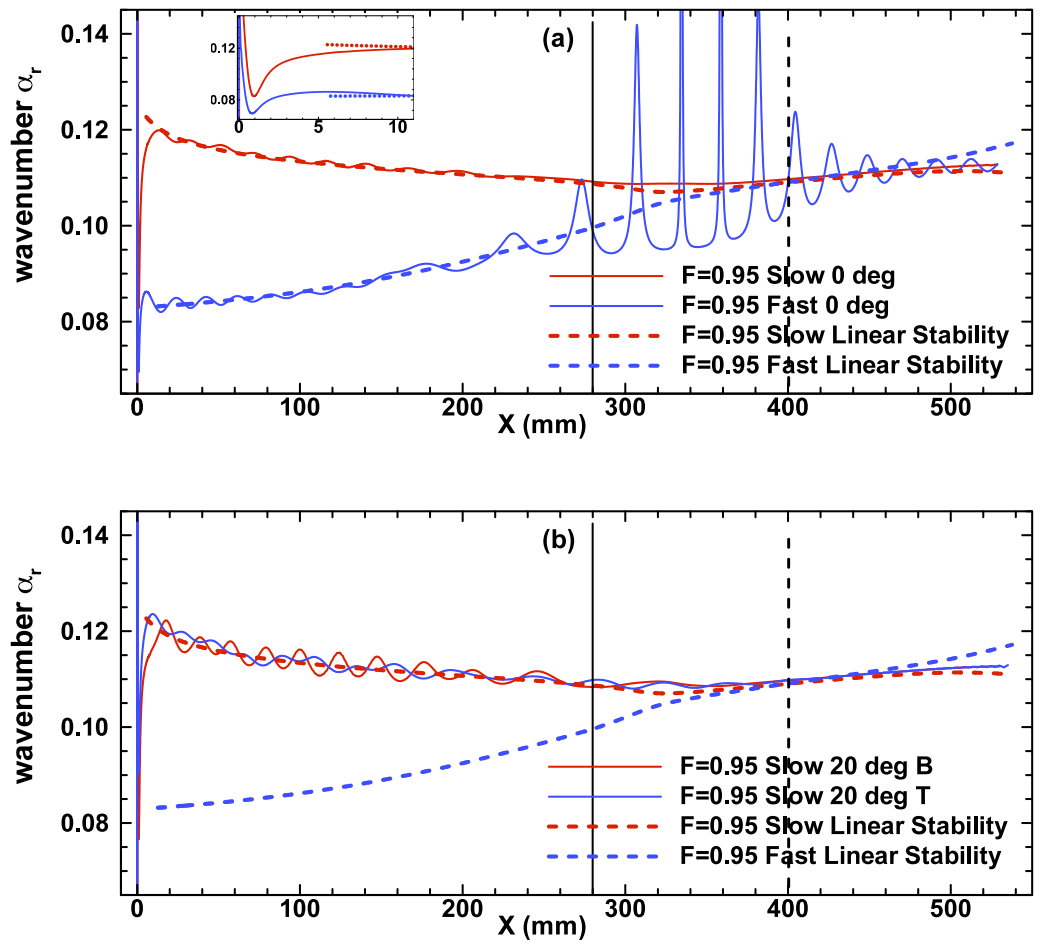

Figure 9. Wavenumber of the pressure fluctuations along the wall generated by two-dimensional acoustic waves at incident angles of 0 and 20 degrees and comparison with the linear stability. $F=0.95 * 10^{-4}$. Solid black line is the location of the continuous spectrum; dotted line is the synchronization point.

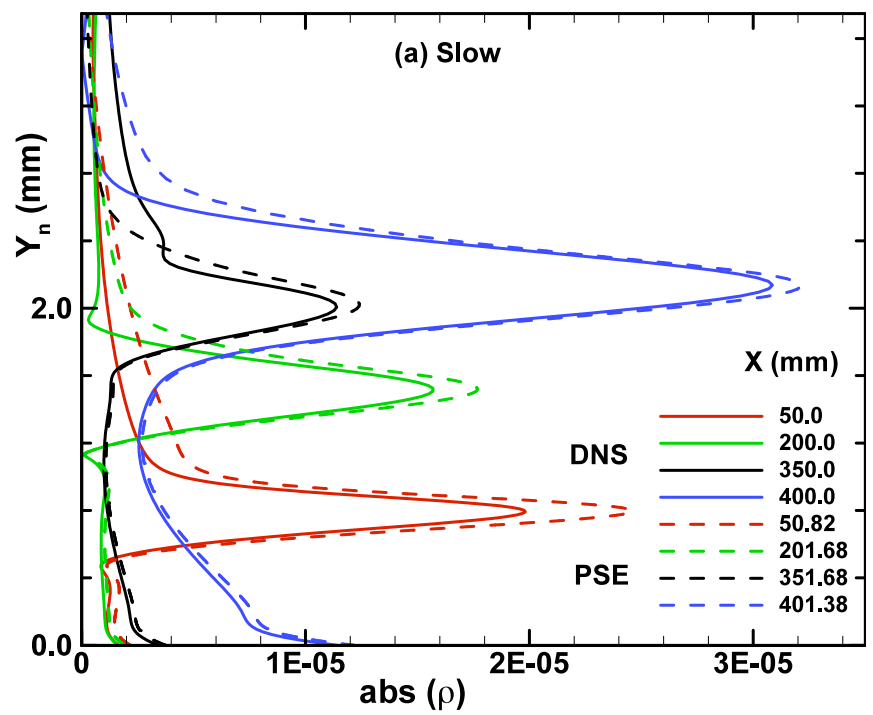

Figure 10. Amplitude distributions for the density at different axial stations generated by the slow acoustic wave at 0 degrees incidence and comparison with the PSE calculations. $F=0.95 * 10^{-4}$. 
(a) $X=200 \mathrm{~mm}$
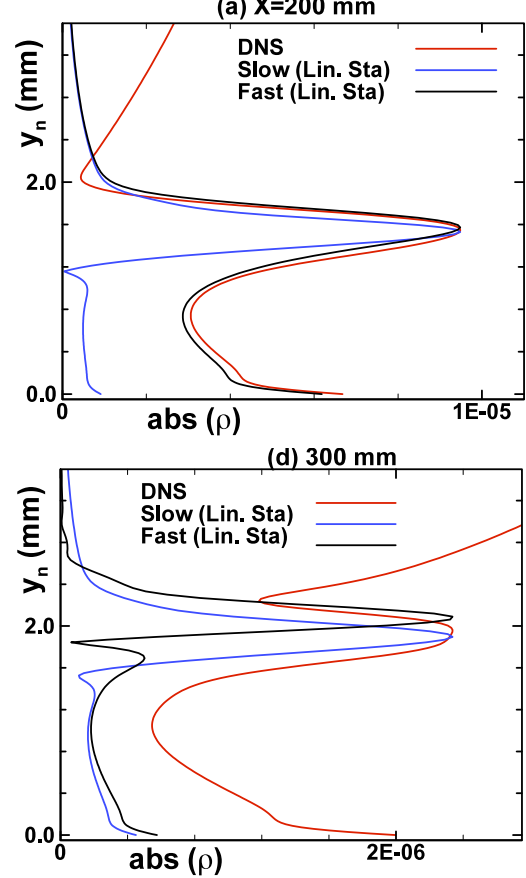

(g) $375 \mathrm{~mm}$

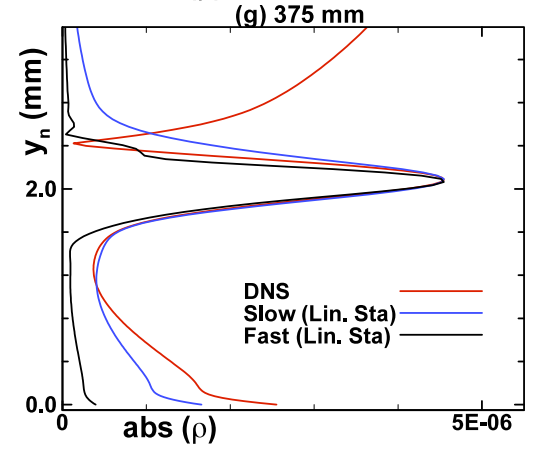

(b) $250 \mathrm{~mm}$

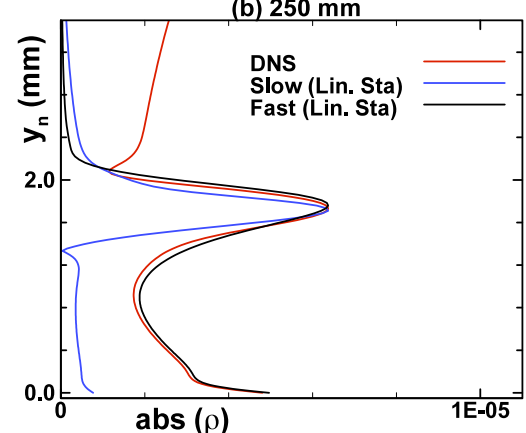

(e) $325 \mathrm{~mm}$

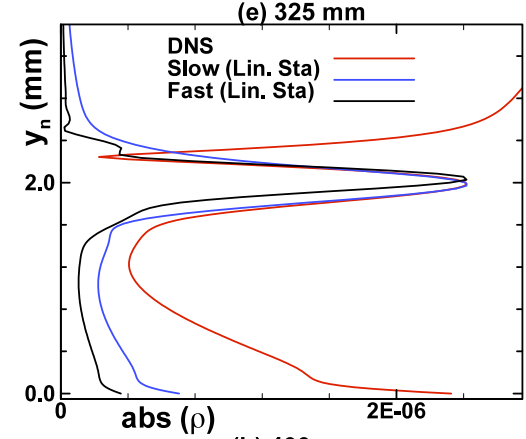

(h) $400 \mathrm{~mm}$

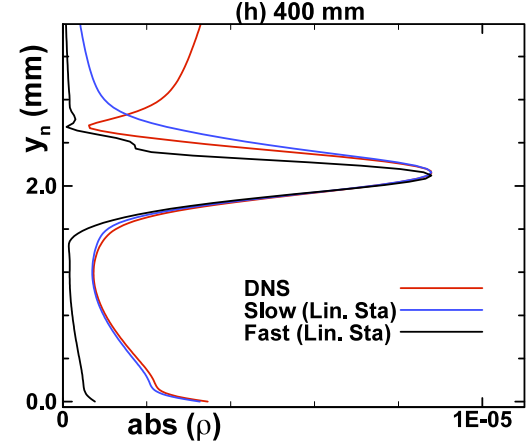

(c) $275 \mathrm{~mm}$

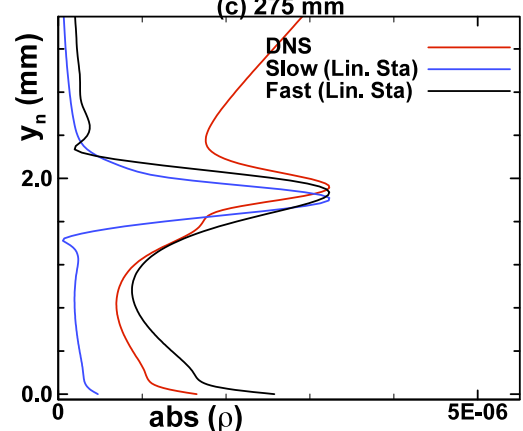

(f) $350 \mathrm{~mm}$
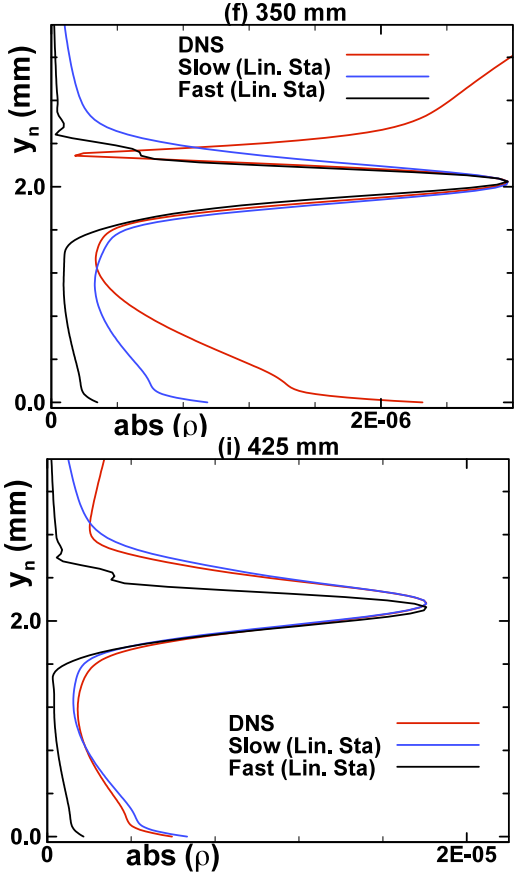

Figure 11. Amplitude distributions for the density at different axial stations generated by the fast acoustic wave at 0 degrees incidence and comparison with the PSE calculations. $F=0.95 * 10^{-4}$. 

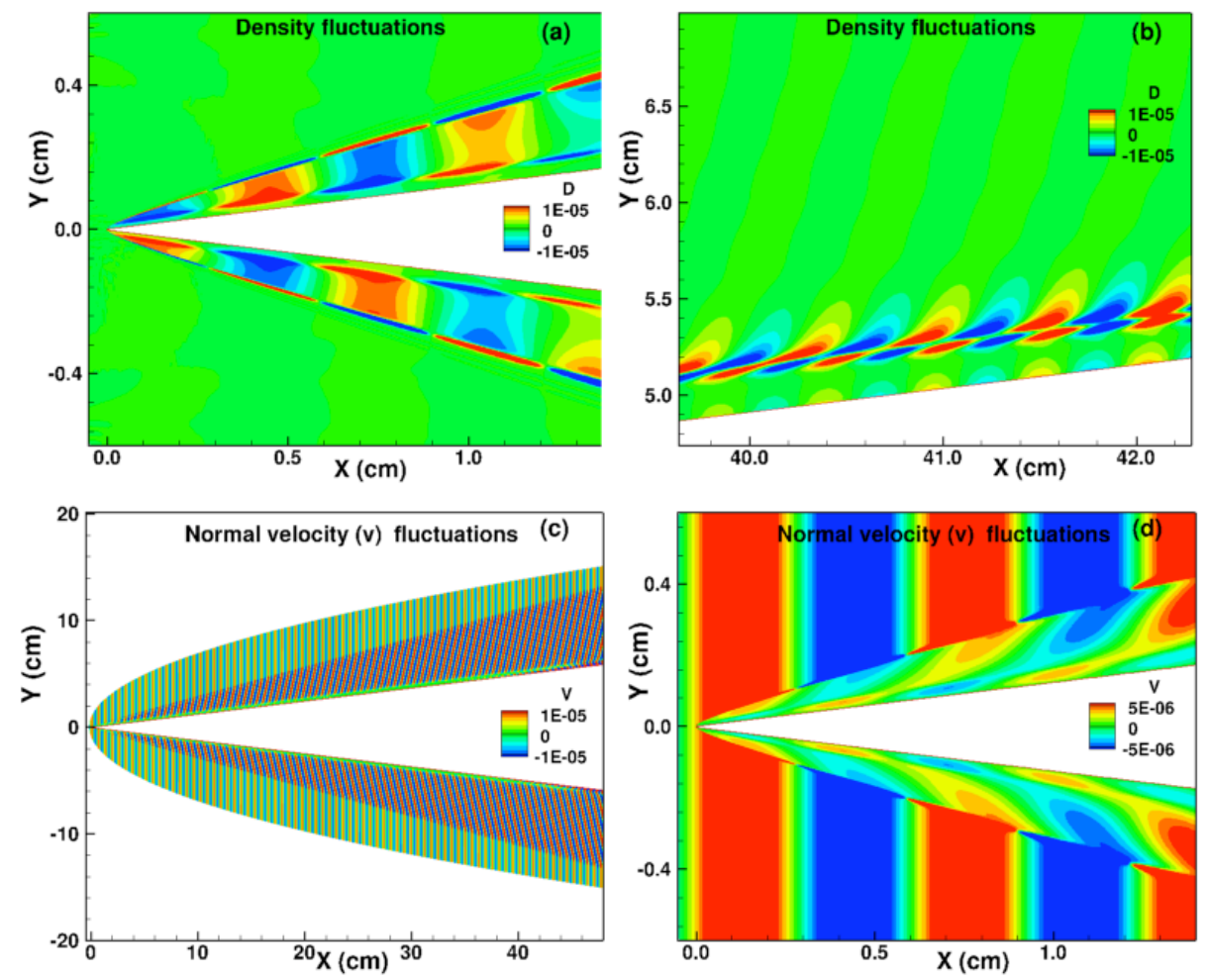

Figure 12. Density and vertical velocity, $v$, fluctuations generated by the interaction of a two-dimensional vortical disturbance with the sharp-tipped wedge. $F=0.95 * 10^{-4}$.
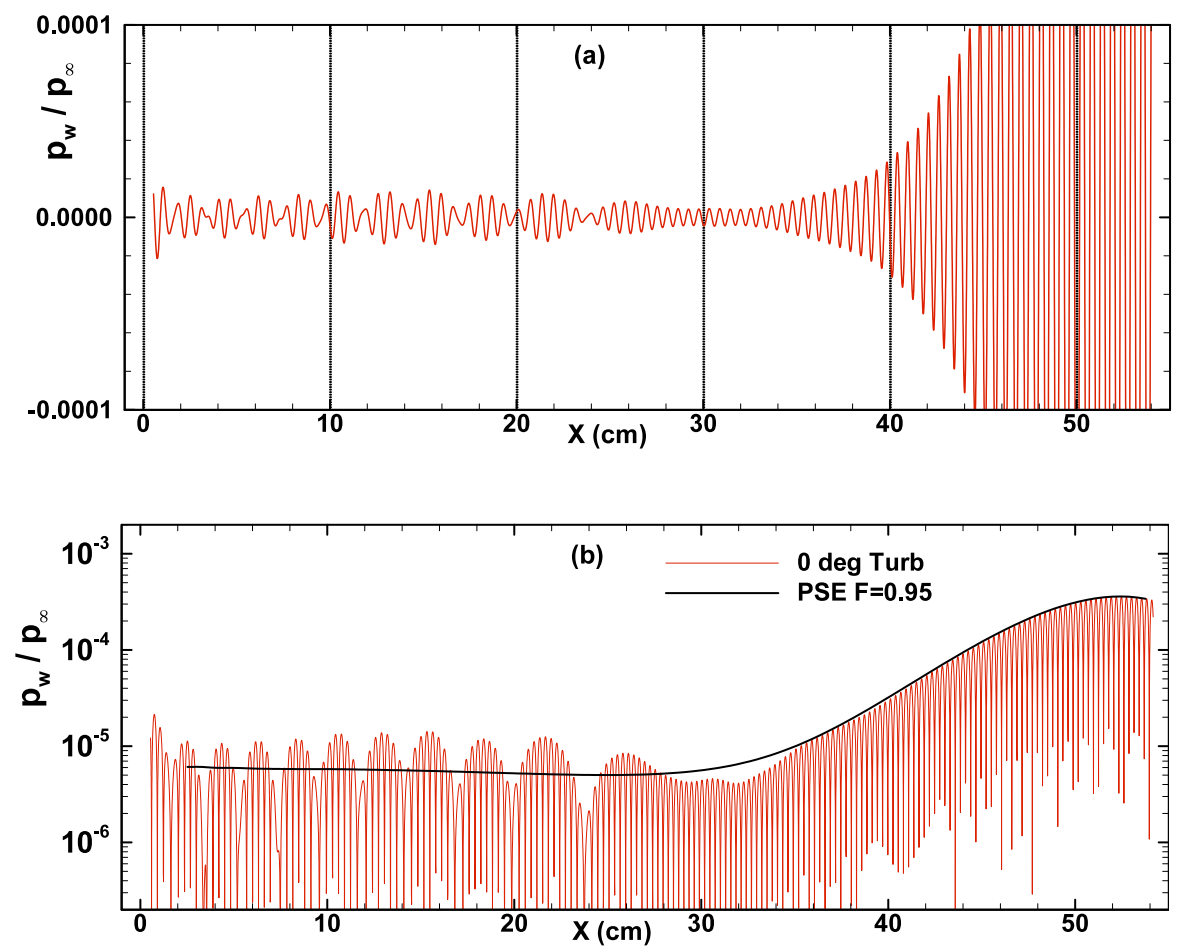

Figure 13. Wall pressure fluctuations generated by the interaction of two-dimensional vortical disturbances with the wedge. $F=0.95 * 10^{-4}$. 


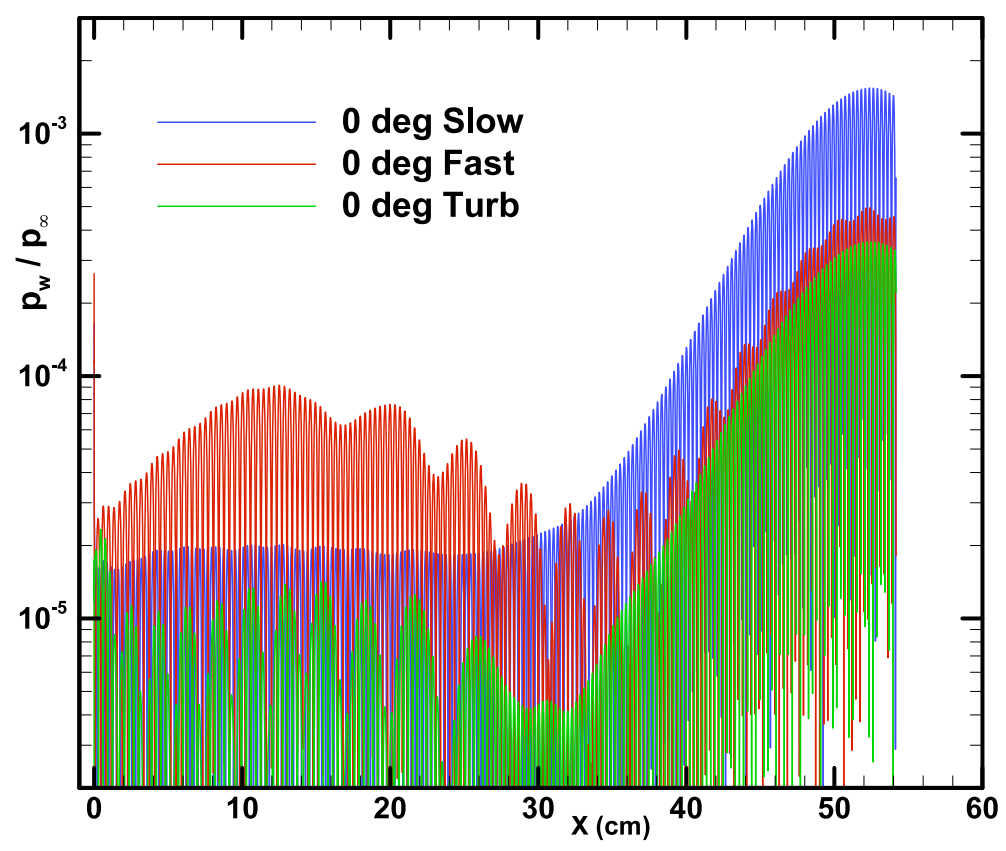

Figure 14. Comparison of the wall-pressure fluctuations along the surface of the wedge generated by the slow and fast acoustic waves, and by the vortical disturbances. $F=0.95 * 10^{-4}$.

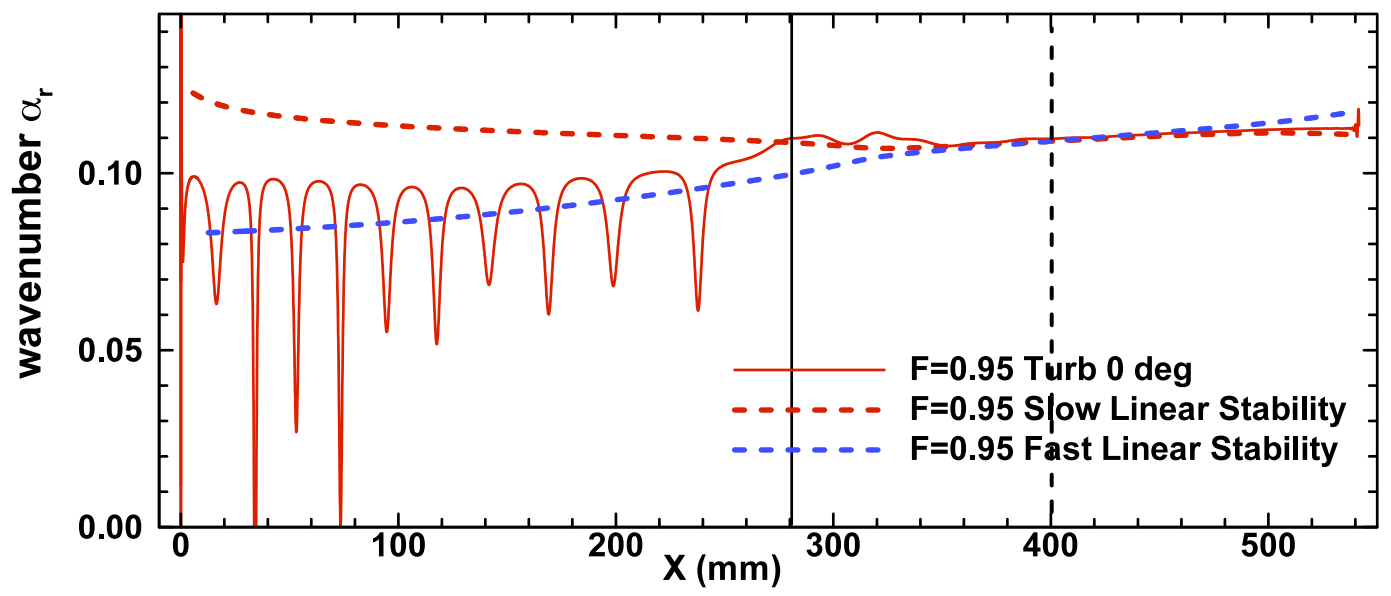

Figure 15. Wavenumber of the pressure fluctuations along the wall generated by the interaction of twodimensional vortical disturbances with the wedge and comparison with the linear stability. Solid black line is the location of the continuous spectrum; dotted line is the synchronization point. $F=0.95 * 10^{-4}$ 
(a) $X=200 \mathrm{~mm}$

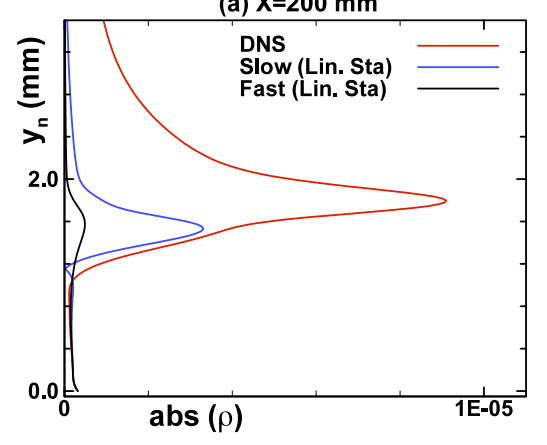

(d) $300 \mathrm{~mm}$

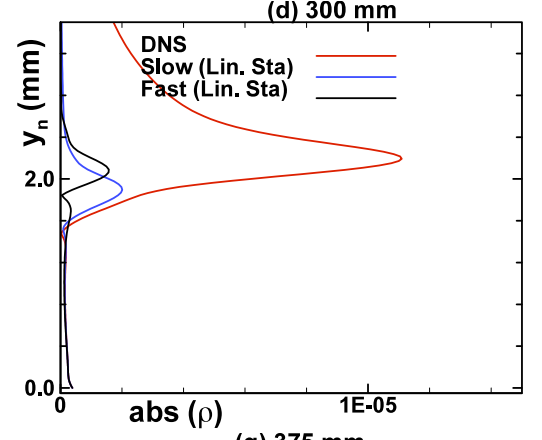

(g) $375 \mathrm{~mm}$

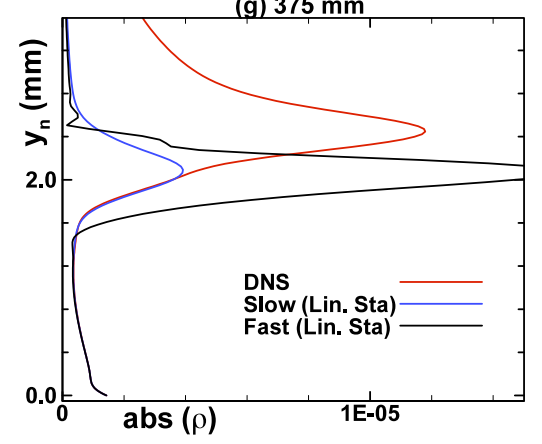

(b) $250 \mathrm{~mm}$

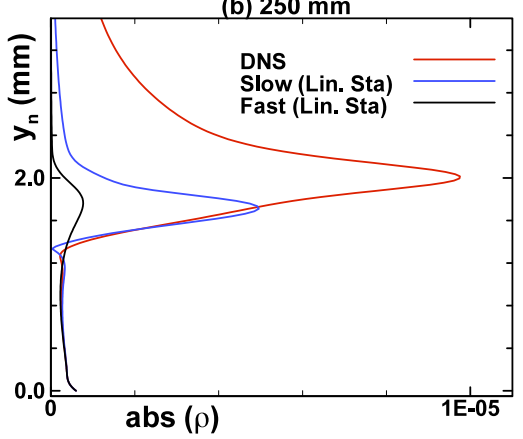

(e) $325 \mathrm{~mm}$

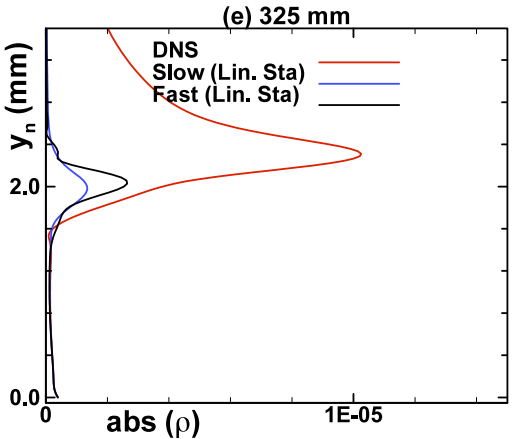

(h) $400 \mathrm{~mm}$

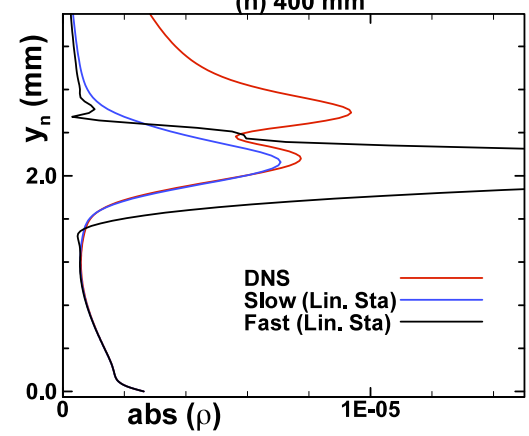

(c) $275 \mathrm{~mm}$
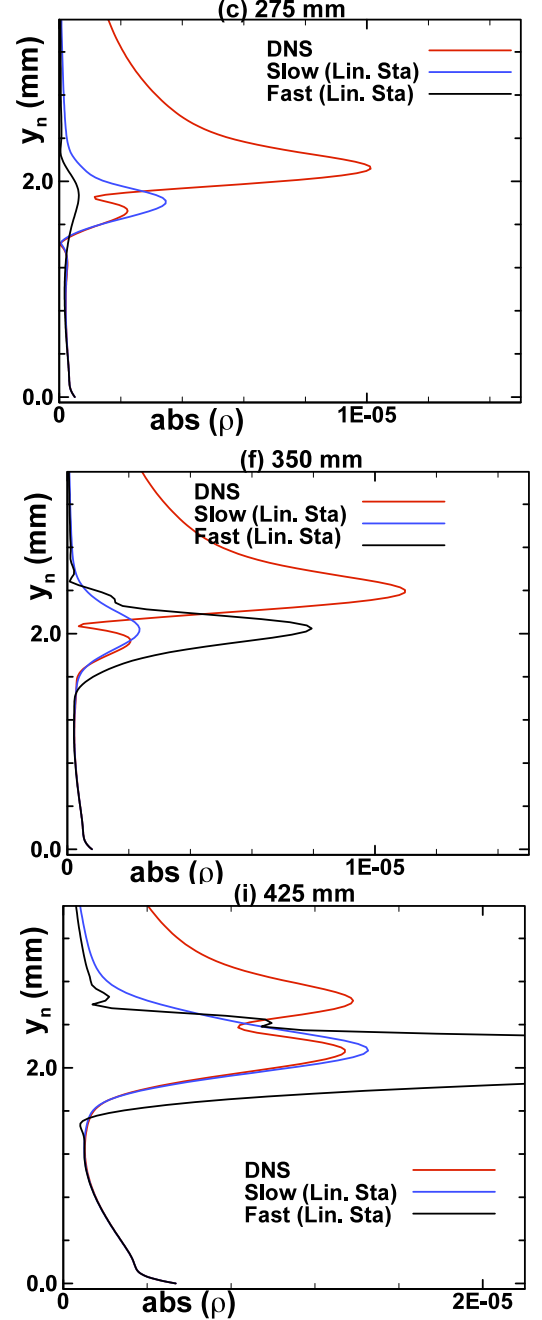

Figure 16. Amplitude distributions for the density at different axial stations generated by the interaction of vortical disturbances with the wedge and comparison with the linear stability calculations. $F=0.95 * 10^{-4}$.
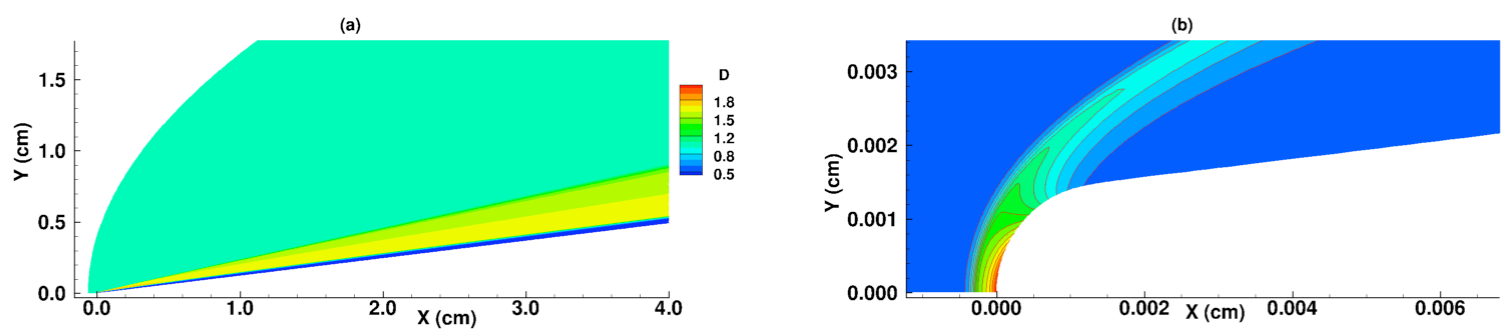

Figure 17. Mean density contours for flow over a 7-degree sharp-tipped cone at Mach 6. 
(a)

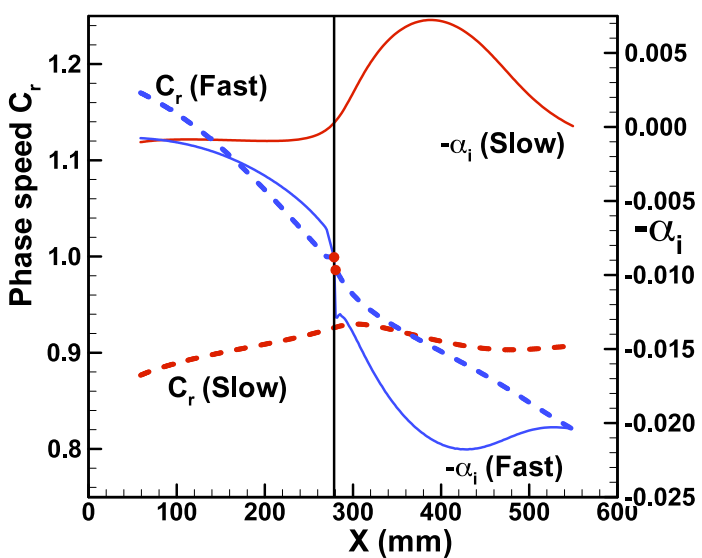

(b)

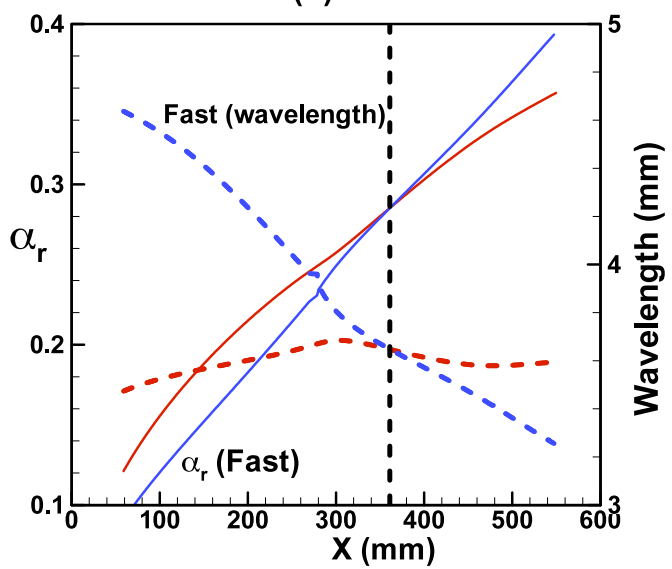

Figure 18. Eigenvalues from the linear stability computations for fast (blue) and slow (red) modes for the frequency $F=1.5 * 10^{-4}$. (a) Phase speed $c_{r}$ and growth rates $-\alpha_{i}$ and (b) wavenumber $\alpha_{r}$ and wavelength.
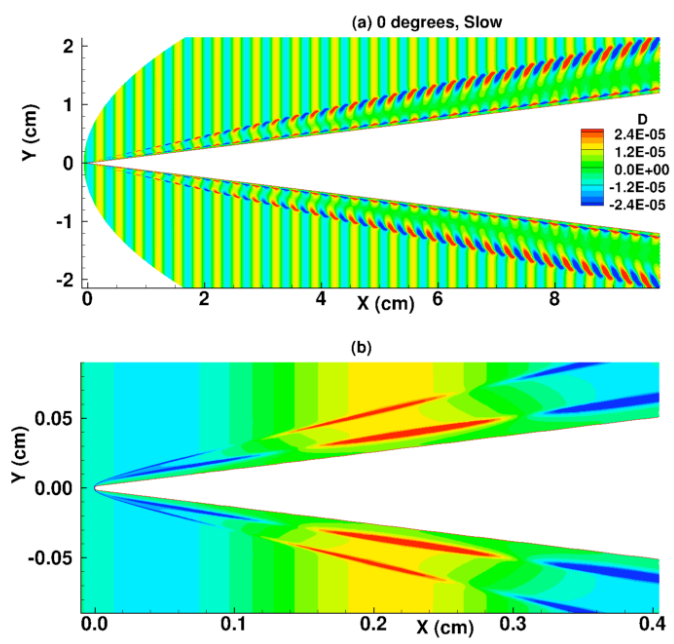
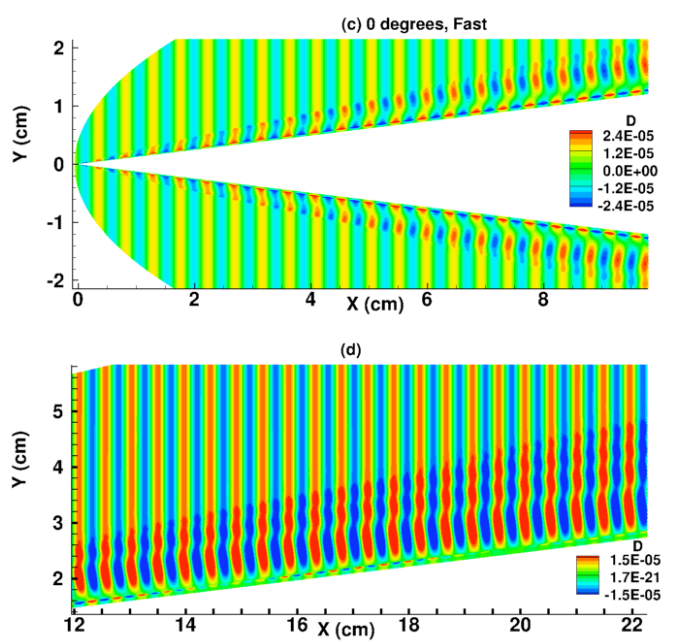

Figure 19. Density fluctuations generated by the interaction of a two-dimensional plane slow and fast acoustic waves with the sharp-tipped cone, incident angle 0 degrees. $F=1.5 * 10^{-4}$. 

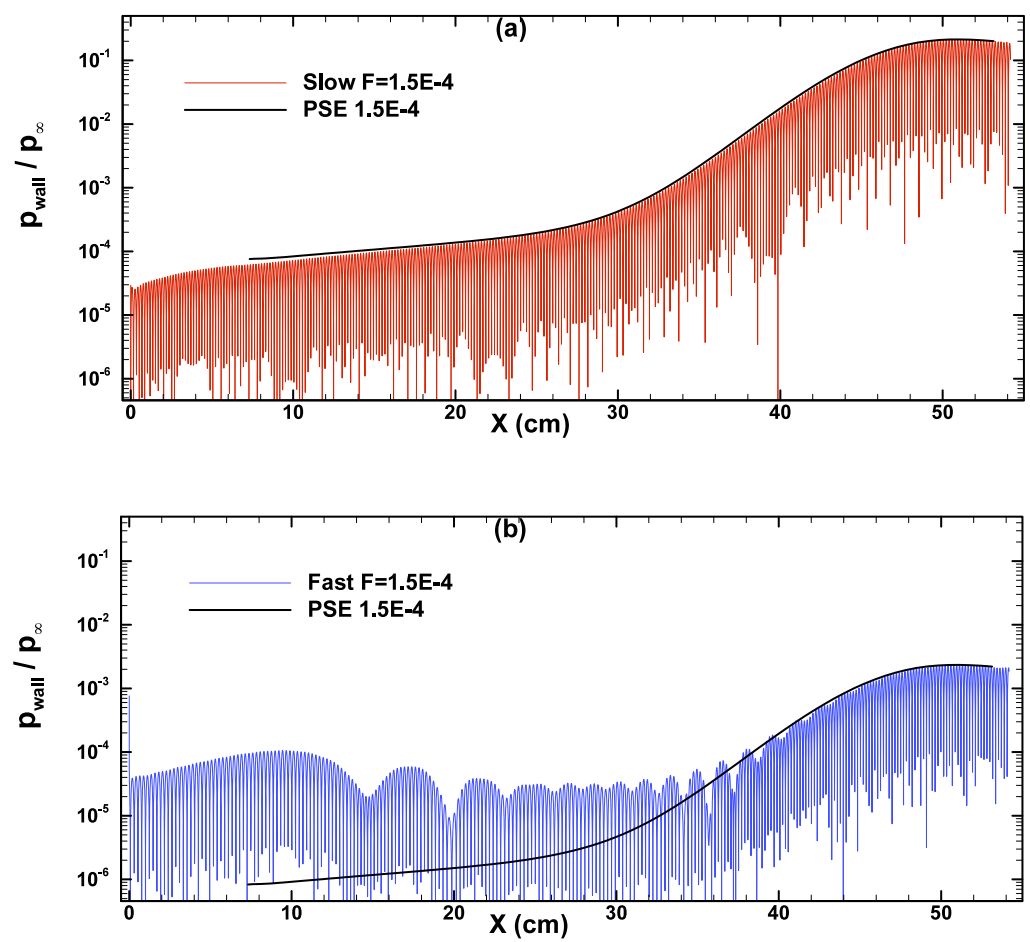

Figure 20. Wall-pressure fluctuations along the sharp-tipped cone obtained from the DNS for twodimensional plane slow and fast acoustic waves.

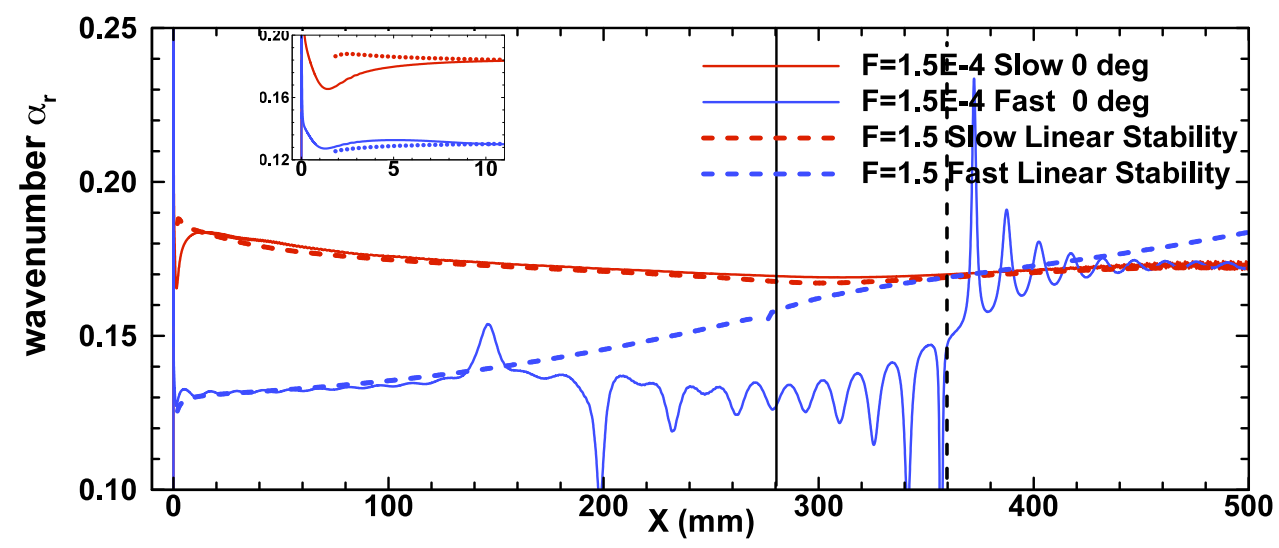

Figure 21. Wavenumber of the pressure fluctuations along the wall and comparison with the linear stability. Solid black line is the location of the continuous spectrum; dotted line is the synchronization point. $F=1.5 * 10^{-4}$. 


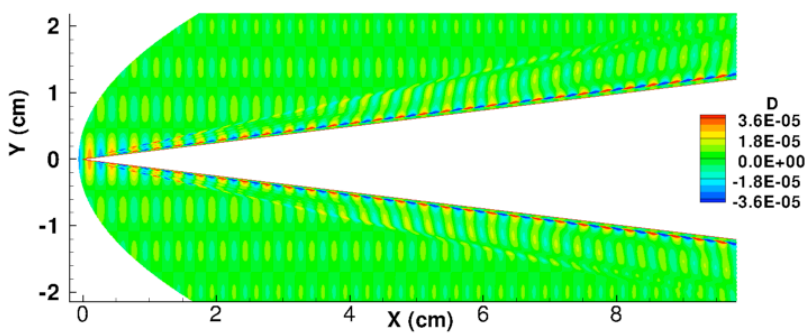

(b)

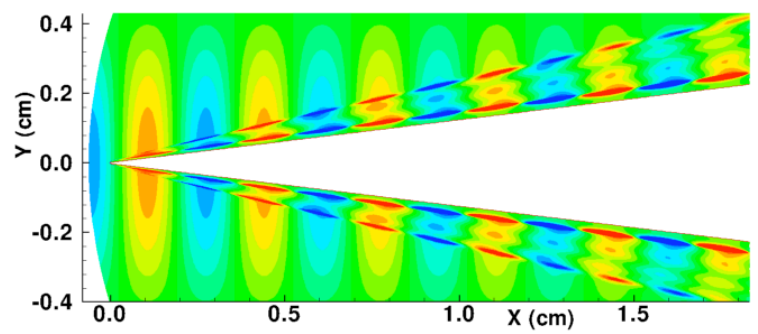

Figure 22. Density fluctuations generated by the interaction of a slow two-dimensional circular acoustic wave with the sharp-tipped cone. $F=1.5 * 10^{-4}$.

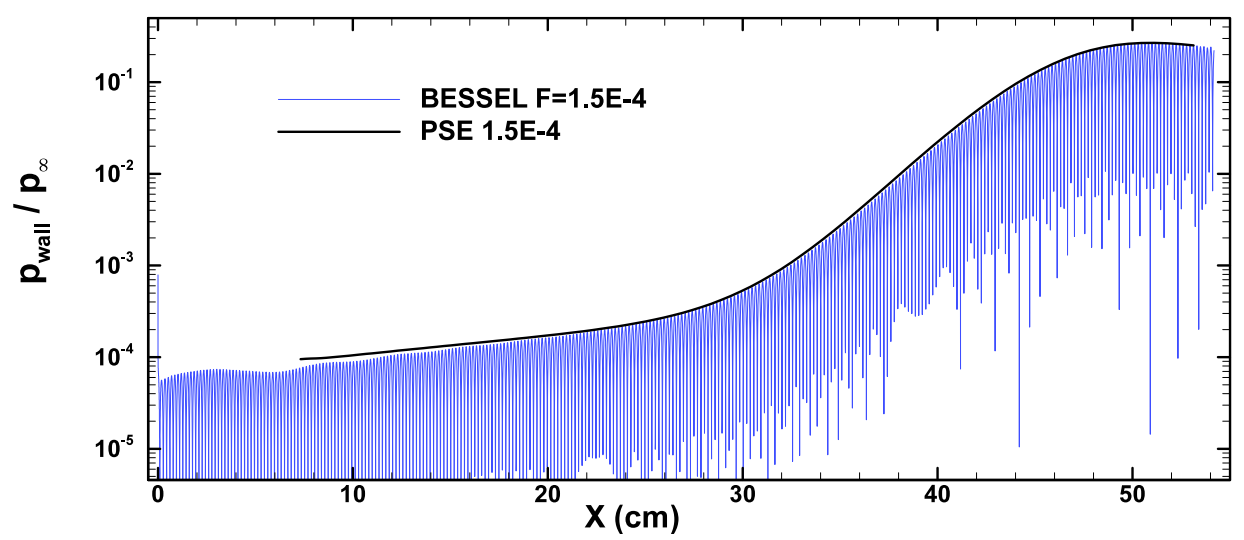

Figure 23. Wall-pressure fluctuations along the sharp-tipped cone obtained from the DNS for a circular acoustic wave.

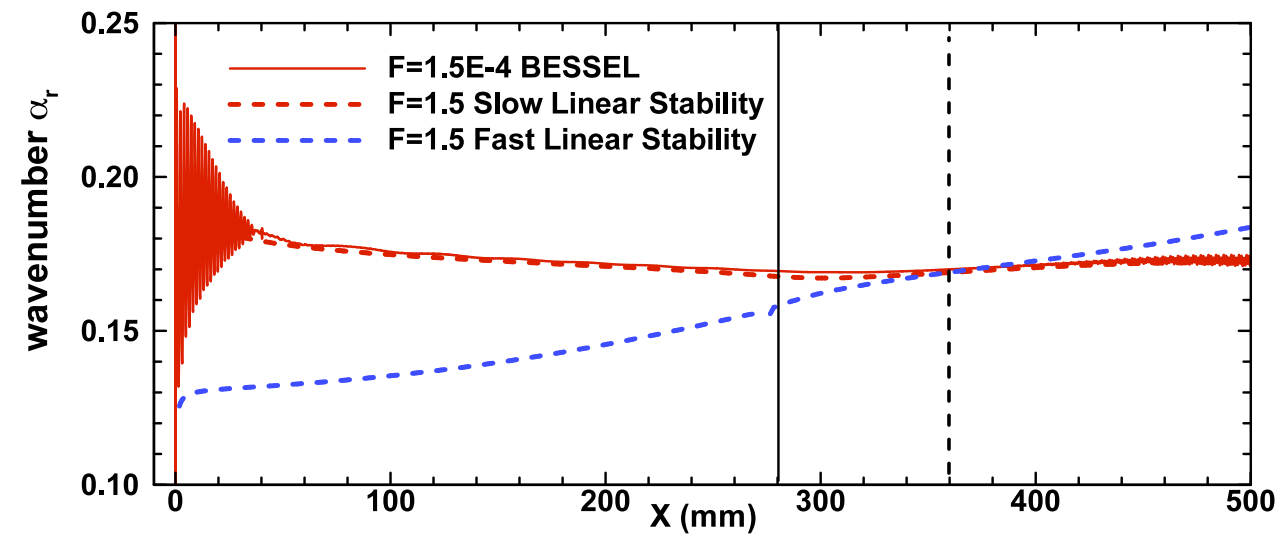

Figure 24. Wavenumber of the pressure fluctuations generated by the interaction of a slow two-dimensional circular acoustic wave with the sharp-tipped cone and comparison with the linear stability. Solid black line is the location of the continuous spectrum; dotted line is the synchronization point. $F=1.5 * 10^{-4}$ 


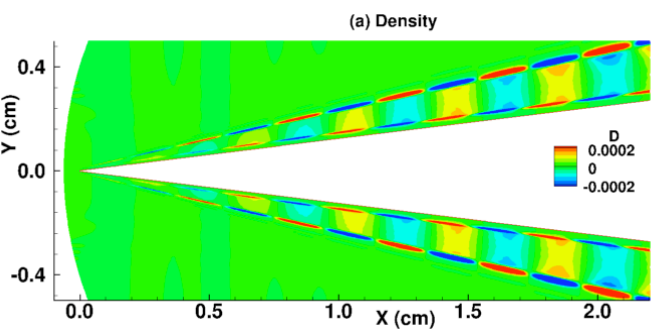

(b)

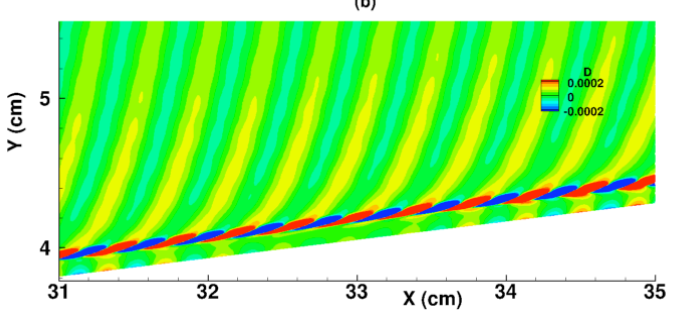

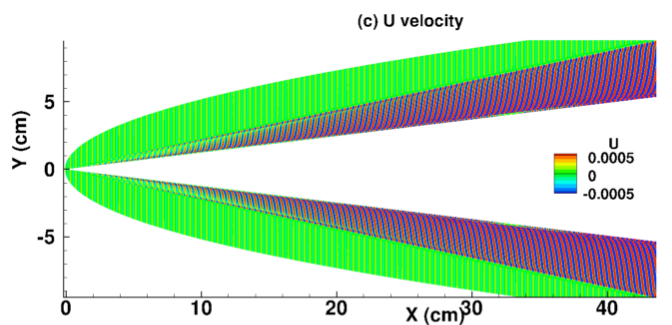

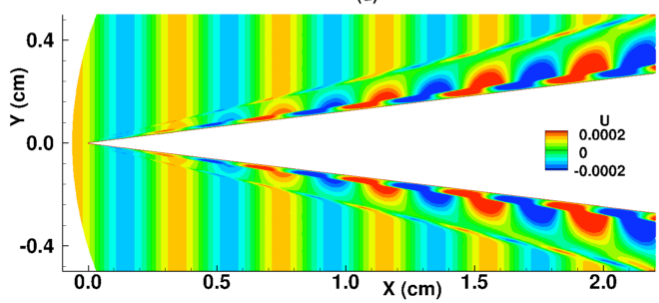

Figure 25. Density $(a, b)$ and u velocity $(c, d)$ fluctuations generated by the interaction of an axi-symmetric vortical disturbance with the sharp-tipped cone. $F=1.5 * 10^{-4}$

(a)

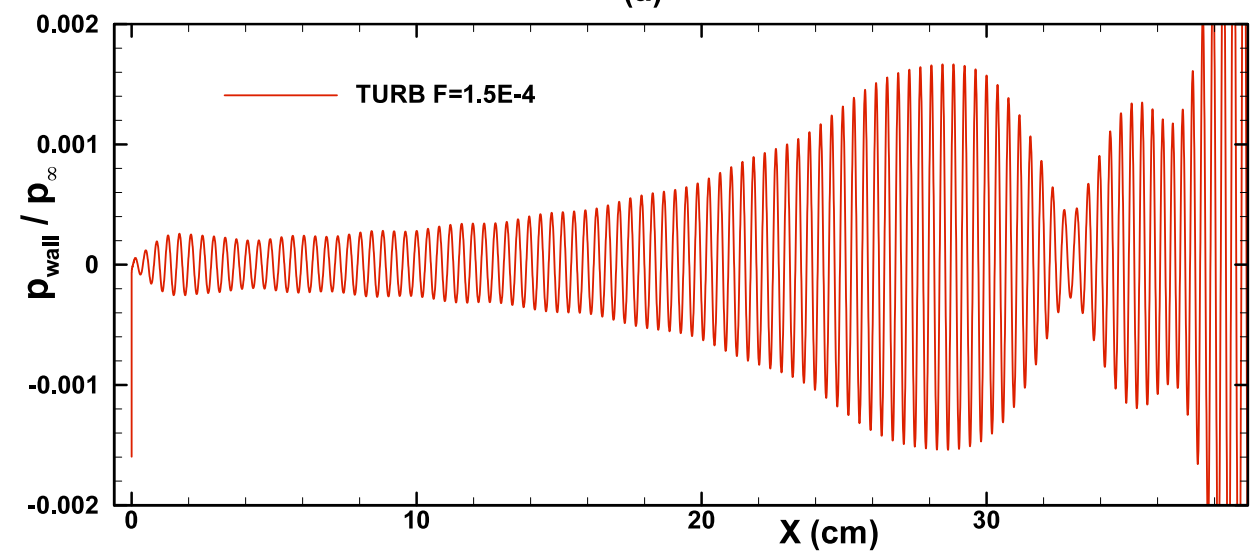

(b)

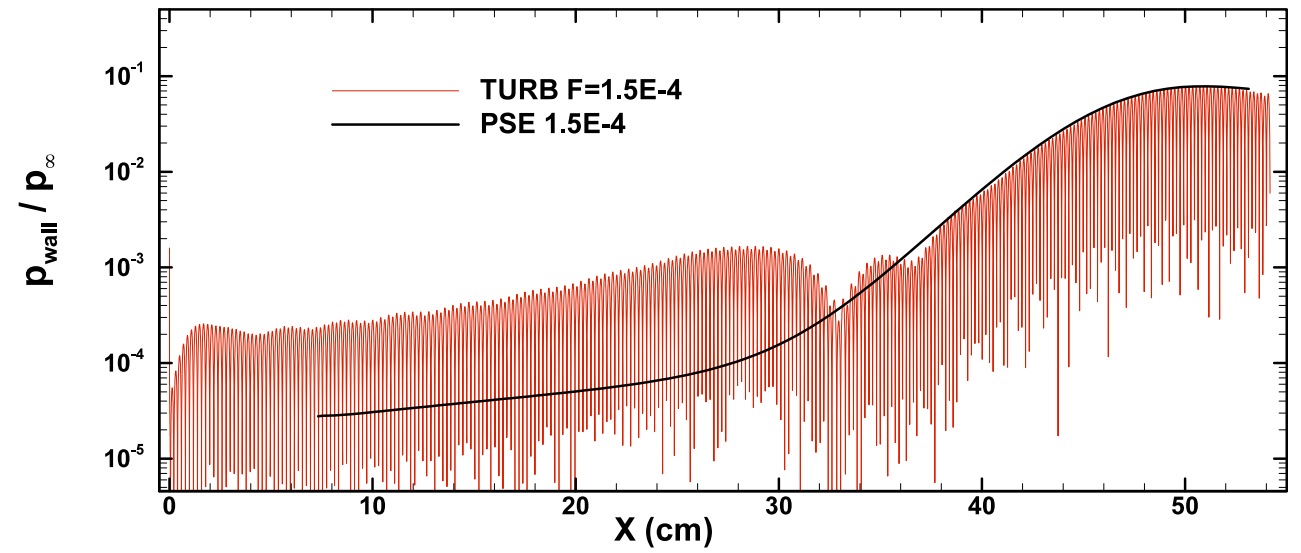

Figure 26. Wall pressure fluctuations generated by the interaction of an axi-symmetric vortical disturbances with the cone (a) in linear scale (b) in log-scale. $F=1.5 * 10^{-4}$. 


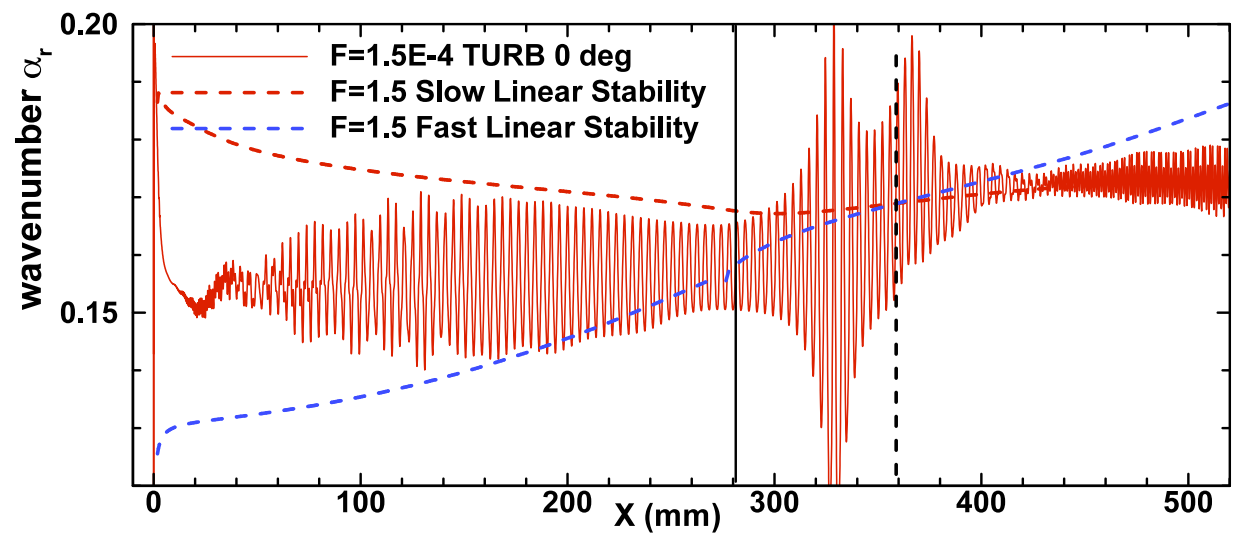

Figure 27. Wavenumber of the pressure fluctuations along the wall generated by the interaction of an axisymmetric vortical disturbance with the sharp-tipped cone and comparison with the linear stability. Solid black line is the location of the continuous spectrum; dotted line is the synchronization point. $F=1.5 * 10^{-4}$ 